\title{
SDF1 Reduces Interneuron Leading Process Branching through Dual Regulation of Actin and Microtubules
}

\author{
Daniel E. Lysko, ${ }^{1}$ Mary Putt, ${ }^{2}$ and Jeffrey A. Golden ${ }^{3}$ \\ ${ }^{1}$ Cell and Molecular Biology Graduate Group, University of Pennsylvania, Philadelphia, Pennsylvania 19104, ${ }^{2}$ Department of Biostatistics and \\ Epidemiology, University of Pennsylvania School of Medicine, Philadelphia, Pennsylvania 19104, and ${ }^{3}$ Department of Pathology and Laboratory Medicine, \\ Children's Hospital of Philadelphia, Philadelphia, Pennsylvania 19104
}

\begin{abstract}
Normal cerebral cortical function requires a highly ordered balance between projection neurons and interneurons. During development these two neuronal populations migrate from distinct progenitor zones to form the cerebral cortex, with interneurons originating in the more distant ganglionic eminences. Moreover, deficits in interneurons have been linked to a variety of neurodevelopmental disorders underscoring the importance of understanding interneuron development and function. We, and others, have identified SDF1 signaling as one important modulator of interneuron migration speed and leading process branching behavior in mice, although how SDF1 signaling impacts these behaviors remains unknown. We previously found SDF1 inhibited leading process branching while increasing the rate of migration. We have now mechanistically linked SDF1 modulation of leading process branching behavior to a dual regulation of both actin and microtubule organization. We find SDF1 consolidates actin at the leading process tip by de-repressing calpain protease and increasing proteolysis of branched-actin-supporting cortactin. Additionally, SDF1 stabilizes the microtubule array in the leading process through activation of the microtubule-associated protein doublecortin (DCX). DCX stabilizes the microtubule array by bundling microtubules within the leading process, reducing branching. These data provide mechanistic insight into the regulation of interneuron leading process dynamics during neuronal migration in mice and provides insight into how cortactin and $D C X$, a known human neuronal migration disorder gene, participate in this process.
\end{abstract}

Key words: cortactin; cytoskeleton; DCX; interneuron; migration; SDF1

\section{Introduction}

Normal cerebral cortical development requires interneurons to migrate dorsally from the ventral ganglionic eminence to integrate with radially migrating projection neurons in the nascent cortical plate (Marín and Rubenstein, 2003). Interneurons must navigate a complex path encountering several choice points. How extracellular guidance factors regulate cytoskeletal proteins to influence interneuron migration is an area of active investigation.

Cell migration requires the orchestration of many intracellular processes culminating in dynamic cytoskeletal restructuring causing membrane protrusion and the movement of cytoplasmic organelles (Ridley, 2011; de Forges et al., 2012). In migrating

\footnotetext{
Received Sept. 11, 2012; revised Feb. 18, 2014; accepted Feb. 20, 2014

Author contributions: D.E.L. and J.A.G. designed research; D.E.L. performed research;D.E.L. and M.P. contributed unpublished reagents/analytic tools; D.E.L. analyzed data; D.E.L. and J.A.G. wrote the paper.

This work was supported by National Institutes of Health grants NS45034 and HD26979. We thank the members of the Golden Laboratory for their helpful comments and support. We are grateful to Mr. George Clement for the meticulous animal husbandry he provided in supported of this work. We thank Dr. Jonathan Raper, Dr. Gaia Colasante, and Dr. Vania Broccoli for the SDF1a expression vectors, Dr. Joseph LoTurco for the DCX expression and RNAI constructs, and Dr. David Solecki for the RFP-Utrophin construct. We thank Dr. Ralf Stumm for the kind gift of the CXCR4 antibody. We thank Alexander Babich in Dr. Janis Burkhardt's laboratory for preparing T-cells. We thank Dr. Stewart Anderson, Dr. Catherine Lee May, Dr. Judith Grinspan, Dr. Eric Marsh, and their labs for sharing lab resources and ideas.

The authors declare no competing financial interests.

Correspondence should be addressed to Jeffrey A. Golden, Department of Pathology, Brigham and Women's Hospital, 75 Francis Street, Boston, MA 02115. E-mail: jagolden@partners.org.

DOI:10.1523/JNEUROSCI.4351-12.2014

Copyright $\odot 2014$ the authors $\quad 0270-6474 / 14 / 344941-22 \$ 15.00 / 0$
}

neurons, significant progress has been made in understanding the cytoskeletal mechanics of nuclear movement (nucleokinesis). Actin/myosin II contraction at the rear and microtubuledependent, dynein-driven nuclear sliding forces combine to propel the nucleus forward (Bellion et al., 2005; Tsai et al., 2007). The microtubule-associated proteins Lissencephaly-1 (LIS1) and doublecortin (DCX) participate in nucleokinesis by coupling dynein, microtubules, and the nucleus together (Tanaka et al., 2004b). Mutations in LIS1 or DCX inhibit normal migration, contributing to the phenotype observed in Type 1 lissencephaly, a brain disorder associated with epilepsy and intellectual disabilities (Pilz et al., 1998; Matsumoto et al., 2001; Vallee et al., 2001; D’Agostino et al., 2002; McManus et al., 2004).

In contrast, we know less about the regulation of the cytoskeleton within the leading process of migrating neurons. Leading process branching allows interneurons to probe the extracellular environment for guidance and change migration direction (Lysko et al., 2011). Understanding leading process cytoskeletal mechanics and regulation is paramount to understanding the neuronal migration defects found in neurodevelopmental disabilities (Métin et al., 2006; Friocourt et al., 2011). Two guidance factors have been shown to regulate interneuron branching: Neuregulin1 (Nrg1) and stromal cell-derived factor-1 (SDF1/ CXCL12; Flames et al., 2004; Li et al., 2008; López-Bendito et al., 2008). Nrg1 is an attractant that promotes branch extension proximal to a point source, while SDF1 acts as a motogen or 
attractant and reduces branching frequency (Li et al., 2008; Martini et al., 2009; Lysko et al., 2011).

Our mechanistic understanding of neuronal branching is informed by studies in axon guidance. A new branch initiated by actin protrusion is then supported by microtubule invasion (Kalil et al., 2000; Dent and Kalil, 2001; Gibson and Ma, 2011). We hypothesize that both actin and microtubules are coregulated by interneuron guidance molecules to tune branching, as interneurons change their branching frequencies as they traverse different zones of tissue (Martini et al., 2009).

We previously found that SDF1 reduces interneuron branching frequency while promoting stream migration (Lysko et al., 2011). How SDF1 regulates the cytoskeleton to reduce interneuron branching is unknown. Here we have identified cortactin as a novel modulator of interneuron branching, and find that SDF1 regulates calpain cleavage of cortactin consolidating actin and reducing branching. SDF1 regulates microtubule organization by allowing DCX to bundle and stabilize microtubules, reducing branching. Our model of SDF1 signaling provides a mechanistic understanding as to how extracellular guidance factors create structural changes in the cytoskeleton to control interneuron branching, ultimately affecting the guidance of migrating interneurons.

\section{Materials and Methods}

Mouse strains. C57BL/6, CD1, or Dlx5/6-Cre-IRES-eGFP mice (Stenman et al., 2003) on a CD1 background and of either sex were used for all experiments. The Institutional Animal Care and Use Committee at the Children's Hospital of Philadelphia, Philadelphia, PA, approved all studies.

Brain explant culture. Brains from embryonic day 14.5 (E14.5) mouse embryos were dissected in ice-cold HBSS (Sigma), embedded in $4 \%$ low melting point agarose (Lonza) in HBSS, and sliced coronally at $250 \mu \mathrm{m}$ thick. One hundred micrometer square explants were cut out of the medial ganglionic eminence (MGE) of the slice and cultured in a 3D extracellular matrix gel on plastic dishes or glass coverslips. The explants were covered with $50 \%$ Matrigel, $50 \%$ collagen $(2 \mathrm{mg} / \mathrm{ml}$; BD Biosciences), and placed at $37^{\circ} \mathrm{C}, 5 \% \mathrm{CO}_{2}$ for $20 \mathrm{~min}$ to gel. Tissue was incubated in DFS media (F12:DMEM w/10\% fetal bovine (FBS) serum, $40 \mu \mathrm{M}$ L-glutamine, and $47 \mathrm{~mm}$ glucose, $\mathrm{P} / \mathrm{S}$ ) for $1 \mathrm{~h}$, then switched to DM media (DMEM w/N2, $36 \mathrm{~mm}$ glucose, and P/S).

T-cell culture. T-cells were isolated from adult C57BL/6 spleen by red blood cell lysis (ACK hypotonic buffer) followed by negative selection using $\alpha$-CD8 (2.43, TIB210; ATCC) and $\alpha$-MHC Class II (M5/114.15.2, TIB120; ATCC), yielding 90\% pure CD4+ T-cells (Huang et al., 2008). T-cells were cultured in DMEM with GlutaMAX, 10\% FBS, $1 \% \mathrm{P} / \mathrm{S}, 1 \times$ nonessential amino acids, $2 \mu \mathrm{l}$ BME, and $25 \mathrm{~mm}$ HEPES at $10 \% \mathrm{CO}_{2}$. T-cells were stimulated on $\alpha$-CD3 (145-2C11)/ $\alpha$-CD28 (PVI; BioXCell) coated plates for $2 \mathrm{~d}$, transferred to uncoated plates to rest for at least $4 \mathrm{~d}$, and cultured in Matrigel:collagen gel within $\mu$-slide chemotaxis 3D chambers (Ibidi) to assess the functionality of SDF1 gradients.

Treatment protocols. Interneurons were exposed to even-field SDF1a by coculturing with COS7 or HEK293T cells in gel cubes coexpressing SDFla and dsRed $300 \mu \mathrm{m}$ from MGE explants within Matrigel:collagen gel (BD Biosciences). COS7 or HEK293T cells were transiently transfected with FuGENE 6 (Roche) $2 \mathrm{~d}$ before pelleting and resuspension in an equal volume of Matrigel to form gel cubes. Stimulation of protein kinase A (PKA) was achieved by treating explants with dibutyryl cyclic adenosine monophosphate (dbcAMP; Sigma). Calpain protease was inhibited with either Calpain Inhibitor III or ALLM (Calbiochem). Actin polymerization was inhibited with Latrunculin B (LatrB; Cayman Chemical), while microtubule polymerization was inhibited with nocodazole (10 nм; Sigma).

SDF1 gradient generation. Interneurons were exposed to a gradient of SDF1 by culturing explants within Matrigel:collagen gel matrix within a chemotaxis chamber ( $\mu$-slide chemotaxis 3D; Ibidi). Control or SDF1- expressing HEK293T gel cubes or recombinant SDF1 (136 nM mSDF1- $\alpha$; PreproTech) were added to the appropriate sides of the chamber to generate a gradient of SDF1 across the explant. Measurement of T-cell chemotaxis was used to assess the functionality and timing of these gradients, as T-cell chemotaxis toward SDF1 is well characterized (Debes et al., 2006; Lin and Butcher, 2006). T-cells were injected into the $\mu$-slide chemotaxis chamber in an identical Matrigel:collagen gel as typically used for interneurons, the gel allowed to harden, then media with control- or SDF1-expressing HEK293T gel cubes added, and cells live imaged immediately for $37.5 \mathrm{~h}$. T-cell chemotaxis was assessed by using the Ibidi Chemotaxis Tool to track 20 cells' migration paths over $1 \mathrm{~h}$ periods throughout the time lapse. Chemotaxis toward the source of SDF1 first occurred at $23 \mathrm{~h}$, was robust by $25-26 \mathrm{~h}$, and persisted until the experiment ended at $37.5 \mathrm{~h}$. SDF1 gradients' establishment and robust chemotaxis were seen at similar times in gradients generated with recombinant SDF1. Interneuron migration experiments involving gradient SDF1 were set up in this manner and imaged starting at $16-20 \mathrm{~h}$ after setup and concluding 36-40 h after setup. MGE explants were generated as above and titrated in cold, fluid Matrigel:collagen to reduce the diameter of the explants allowing explants to be loaded into chemotaxis chambers within fresh Matrigel:collagen.

Immunocytochemistry. Interneurons were fixed at $37^{\circ} \mathrm{C}$ for $12 \mathrm{~min}$ in $4 \%$ paraformaldehyde in $1 \times$ PBS with 60 mм PIPES, 25 mm HEPES, 8.1 mM EGTA, and $4 \mathrm{~mm} \mathrm{MgSO}_{4}$. Tubulin was labeled with one of the following antibodies: DM1A ( $\alpha$-tubulin; Sigma), Tuj1 (Neuronal CIII $\beta$-tubulin; Covance), YL1/2 (Tyr- $\alpha$-tubulin; Millipore), or 6-11B-1 (acetylated- $\alpha$ tubulin, Sigma). CXCR4 was labeled with rabbit polyclonal anti-CXCR4 (1181), a kind gift from Dr. R. Stumm (University Hospital Jena, Friedrich-Schiller University, Germany). Microtubule-associated proteins (MAPS) were labeled with the following antibodies: DCX;C-18/ 8066 (Santa Cruz Biotechnology), APC;AP-7/CC-1 (Calbiochem), Tau; T49 (a kind gift from Dr. V. Lee, University of Pennsylvania), and MAP2, MAB378 (Millipore). Actin was labeled with Alexa Fluor 594 phalloidin (Invitrogen). Cortactin was labeled with the 4F11 antibody (Millipore). Spectrin was labeled with the $2122 \mathrm{~S}$ antibody ( $\alpha$-fodrin; Cell Signaling Technology). Proteolyzed spectrin was labeled with Ab37 (a kind gift from Dr. R. Siman, University of Pennsylvania).

Expression constructs. SDF1a expression plasmids were kindly provided by Drs. J. Raper (University of Pennsylvania) and V. Broccoli (San Raffaele Scientific Institute, Italy). DCX-RNAi and DCX plasmids were kindly provided by Dr. J. LoTurco (University of Connecticut). pCAGDCX S47A was created by site-directed mutagenesis using two sets of PCR primers with overlapping mutagenic primers (5' GGC AAA GAA TTC ATG GAA CTT GAT TTT GGA C $3^{\prime}$ and $5^{\prime} \mathrm{ctt} \mathrm{ctt}$ ggc ctt ctt ctc att ggc taa tgc ctg caa ggt tct ggt $\left.\mathrm{t} 3^{\prime}\right)$ and $\left(5^{\prime}\right.$ aac cag aac ctt gca ggc att agc caa tga gaa gaa ggc caa gaa g $3^{\prime}$ and $5^{\prime}$ TAT ATG CGG CCG CTC ACA TGG AAT CGC CAA GT $3^{\prime}$ ), inserted into pCAG-EN and fully sequenced through the expressed coding area. pEGFP-C1-cortactin from Dr. A. Huttenlocher (University of Wisconsin) was acquired via Addgene. Expression in interneurons was low, so we used this plasmid as a template to create pCAG-GFP-cortactin. pCAG-RFP-Utrophin was a kind gift from Dr. D. Solecki (St. Jude Children's Research Hospital, Memphis TN).

Microscopy. Speed and branching analyses were performed on timelapse images acquired at $10 \times$ magnification and at $10 \mathrm{~min}$ intervals for $8-14 \mathrm{~h}$ on a Nikon TE300 microscope equipped with an Okolab environmental chamber $37^{\circ} \mathrm{C}, 5 \% \mathrm{CO}_{2}$. Calpain activity was measured by using the fluorescent CMAC peptidase substrate, t-BOC-LeuMet (t-Boc; Invitrogen) according to previously published methods (Mingorance-Le Meur and O'Connor, 2009). Interneurons were loaded for $10 \mathrm{~min}$ and imaged at $20 \times$ using a DAPI filter cube. Cortactin or DCX overexpressing or knockdown neurons were imaged at $10 \times$ magnification $(2 \times$ zoom $)$ at several $Z$ positions on an Olympus FV10i confocal microscope, $37^{\circ} \mathrm{C}, 5 \% \mathrm{CO}_{2}$. To obtain higher spatial and time resolution as in the branch lifetime analysis, images were acquired at $60 \times$ magnification and at 2 min intervals for 8-12 h on an Olympus FV10i confocal microscope. Immunolabeled interneurons were imaged at $60 \times$ magnification $(2 \times$ zoom $)$ with optimal $Z$ stacks on an Olympus FV10i confocal microscope. 

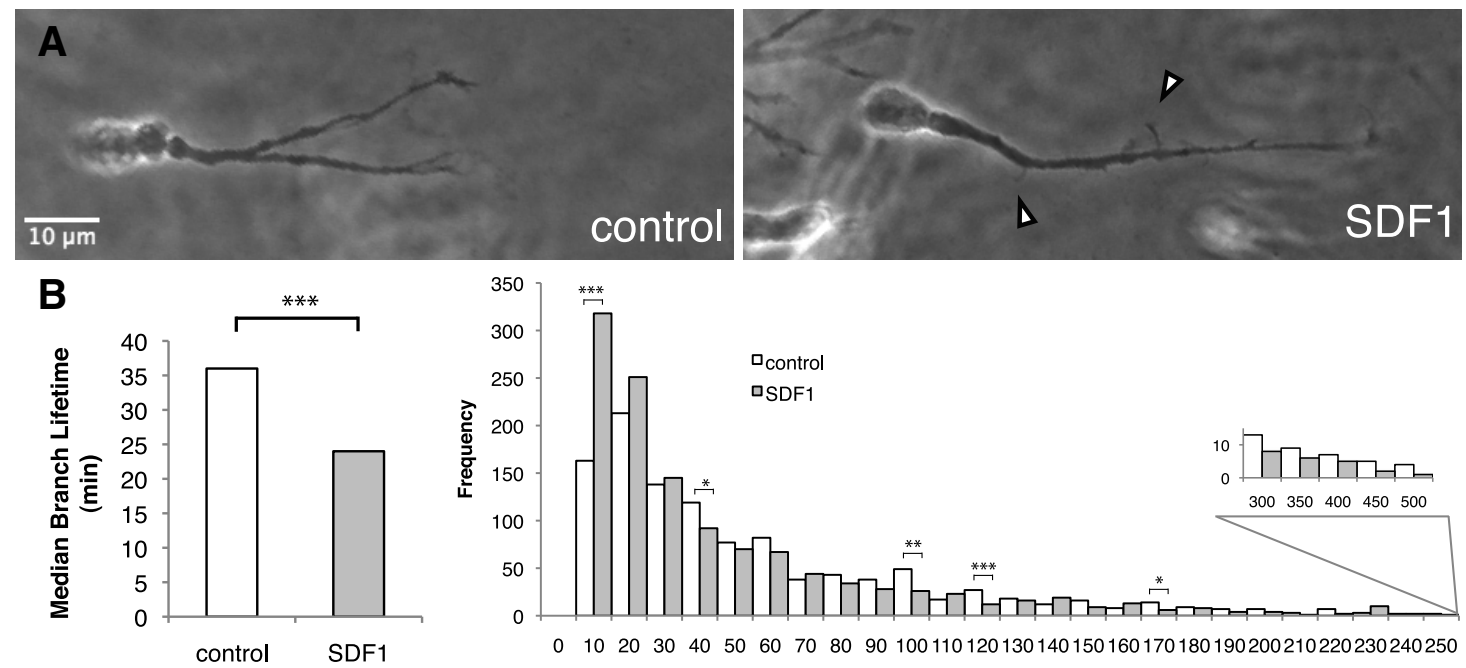

$0 \quad 10203040506070 \quad 8090100110120130140150160170180190200210220230240250$
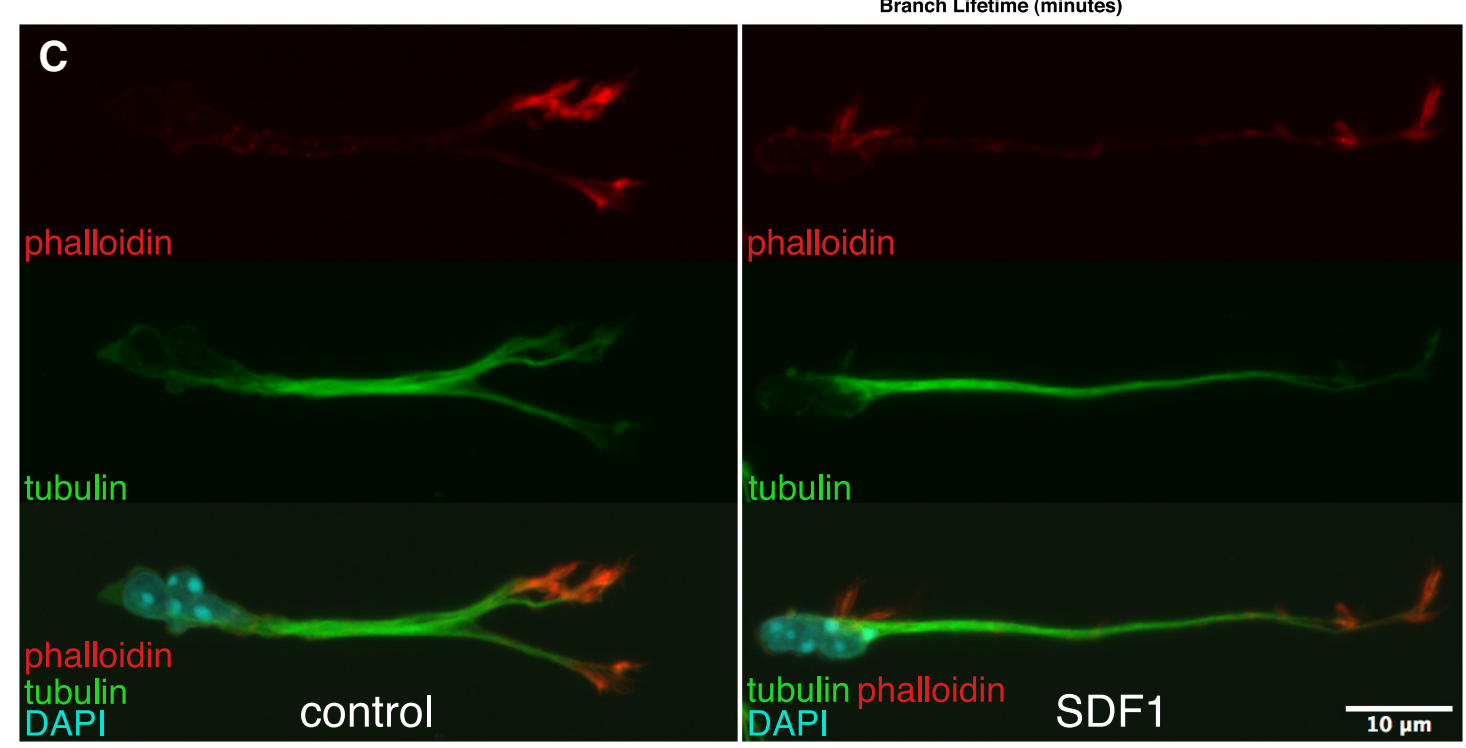

Figure 1. SDF1 decreases branch lifetime and changes actin and microtubule networks. $A$, High-resolution images of migrating interneurons under control conditions or treated with SDF1 (frames from Movie 1). Interneurons treated with SDF1 have many small short-lived branches decorating the main leading process (arrowheads). B, Quantification of branch lifetime shows significantly lower median branch lifetime in interneurons treated with SDF1 ${ }^{* * *} p<0.001$, Wilcoxon rank sum test). Analysis of the distribution of branch lifetimes shows significantly more short-lived branches and fewer long-lived branches in interneurons treated with SDF1 ( ${ }^{* * *} p<0.001,{ }^{* *} p<0.01,{ }^{*} p<0.05, \chi^{2}$ test; $n=75$ cells for each condition, 3 independent experiments). At the highest branch lifetimes, we do not see enough branches for the difference between control and SDF1 to reach significance, but we note that the trend of SDF1 reducing branch lifetime remains consistent. C, Control-and SDF1-treated interneurons labeled for F-actin in red (phalloidin), microtubules in green ( $\alpha$-tubulin), and nuclei in blue (DAPI). SDF1 treatment consolidates F-actin at the leading process tip and consolidates microtubules within the leading process. Scale bars, $10 \mu \mathrm{m}$.

Quantification. Interneuron average speed was measured by recording cell soma position in each frame using the Manual Tracking plugin in ImageJ (National Institutes of Health, NIH; http://rsb.info.nih.gov/ij/) and averaging the speed from each frame in the interval of analysis. Interneuron branching was recorded manually. Interneuron length was measured in frames from time-lapse movies in ImageJ. Cortactin- and phalloidin-positive leading process tip area was quantified in images of cortactin- or phalloidin-stained interneurons using the wand tool in ImageJ. Calpain activity, measured by $\mathrm{t}$-Boc fluorescence, was quantified by measuring gray-value intensity in the cell soma with ImageJ. Directionality analysis of interneuron migration paths was performed using the Chemotaxis Tool plugin for ImageJ (Ibidi; http://ibidi.com) on interneurons migrating at least $100 \mu \mathrm{m}$ from origin.

Statistical analysis. Statistical analysis was performed using Microsoft Excel or R (http://www.r-project.org/) with plugin R-commander. If data appeared normally distributed, we asked whether there were differences between groups using multiway ANOVA followed by post hoc tests adjusted for multiple comparisons using a Bonferroni correction. Branching data appeared slightly non-normally distributed, so a similar

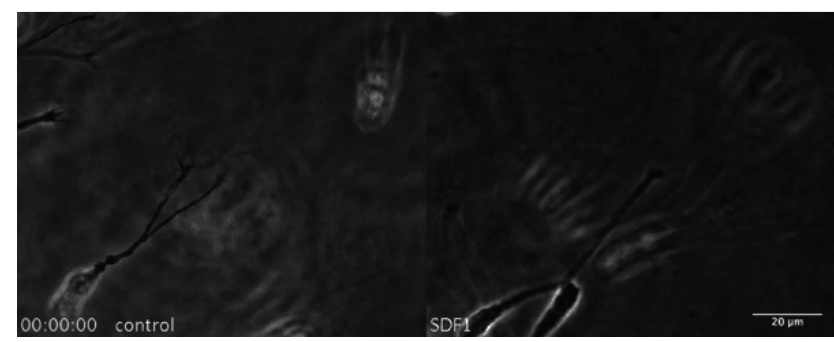

Movie 1. SDF1 decreases branch lifetime. Control- and SDF1-treated interneurons migrating through collagen-Matrigel gel. Interneurons have an extremely dynamic leading process, with many small filopodial protrusions. Control, Control interneurons generate long-lived branches. SDF1, Interneurons treated with SDF1 have fewer stable, long-lived branches and more short-lived branches. $60 \times$ time-lapse phase microscopy, 1 frame/2 min. Scale bar, $20 \mu \mathrm{m}$. 
approach was used, but in these cases a generalized linear model with a Poisson distribution and a term to correct for overdispersion was used to fit the data, yielding an overall test of significance based on analysis of deviance. Differences in interneuron branch lifetime distribution between control- and SDF1-treated interneurons were analyzed using a $\chi^{2}$ test. If a difference was found between the populations, control and treated values for each bin were independently analyzed using two by two contingency tables.

\section{Results}

\section{SDF1 decreases branch lifetime}

SDF1 is one of the few known guidance factors migrating interneurons use to navigate the cerebral cortex during brain development. SDF1 is expressed along the two main streams of migration in the developing cortex: in the meninges and in the subventricular/intermediate zones (SVZ/IZ), where it affects the timing of cortical plate invasion (Stumm et al., 2003, 2007; Li et al., 2008; López-Bendito et al., 2008). We have previously shown that SDF1 affects the timing of cortical plate invasion by reducing branching frequency (Lysko et al., 2011); we next wanted to define how SDF1 signaling regulatesbranchingbehaviorand cytoskeletalmodifying proteins.

Neuronal branching can be divided into steps consisting of branch initiation, growth, and stabilization. A membrane protrusion forms first; the protrusion then grows in size forming a branch. If the branch persists a stable branch has formed. We first sought to understand how SDF1 reduces branching either by decreasing branch initiation, growth, or stabilization. Interneurons migrating out of medial ganglionic eminence explants in $3 \mathrm{D}$ culture were imaged with high spatial and temporal resolution to measure the number and lifetime of membrane protrusions. The interneurons' membrane was quite dynamic and many small, thin protrusions were observed frequently at the tips of the leading process and less frequently along the leading process shaft (Fig. 1A, Movie 1). These data are consistent with our previous observations that leading process splitting (bifurcation at the tip of the leading process) generates $\sim 20 \%$ more branches than interstitial branching (collateral branches that grow out from an established leading process shaft; Lysko et al., 2011). Many protrusions collapse, but protrusions that are stabilized grow and can become new branches that may ultimately change the direction of migration. In SDF1-treated interneurons, many small protrusions are
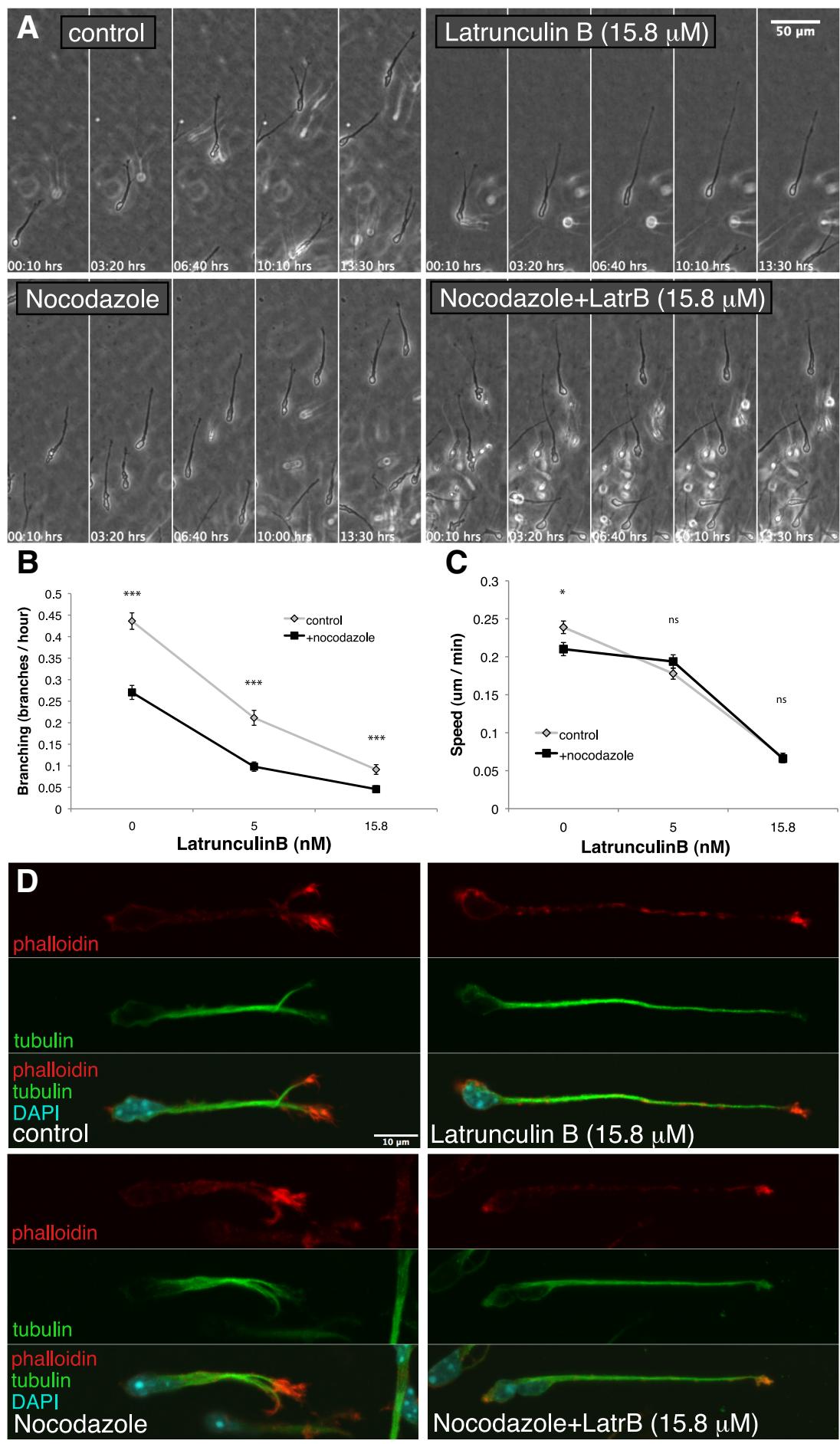

Figure 2. Depolymerizing actin and/or microtubules in migrating interneurons generates distinct migratory behaviors and morphologies. A, Frames from the time-lapse sequence of Movie 2 showing leading process dynamics during actin and microtubule depolymerization. LatrB treatment of migrating interneurons causes a dose-dependent cyclical extension and collapse of leading process branches resulting in an overall reduction in branching and speed $(\boldsymbol{A}, 15.8 \mathrm{~nm}$ LatrB only; Movie 2, both 5 and 15.8nm LatrB). Low-dose nocodazole (10 nм) treatment inhibits branching, generating a brushy leading process appearance, but only modestly decreases speed. LatrB in combination with nocodazole further reduces branching and motility. $B, C$, Quantification of branching and speed in interneurons treated with $L a t r B$ and/or nocodazole. LatrB reduces branching in a dose-dependent manner. Low-dose nocodazole alone also inhibits branching. LatrB in combination with nocodazole further reduces branching ( \pm SEM; $p<0.001$, analysis of deviance, ${ }^{* * *} p<0.001$, post hoc test). LatrB severely inhibits migration in a dose-dependent manner. Nocodazole alone causes a more modest decrease in speed, but in combination with LatrB speed is either slightly increased or not affected depending on the dose ( $\pm \mathrm{SEM} ;{ }^{*} p<0.05, t$ test). Post hoc and $t$ tests are nocodazole treated compared with control/LatrB. $\boldsymbol{D}$, Interneurons treated with LatrB and/or nocodazole labeled for F-actin in red (phalloidin), microtubules in green ( $\alpha$-tubulin), and nuclei in blue (DAPI). LatrB alone or in combination with nocodazole results in a marked consolidation of the F-actin domain at the leading process tip. Nocodazole alone creates a brushy, splaying leading process, in contrast to SDF1 treatment. Scale bars: $\boldsymbol{A}, 50 \mu \mathrm{m} ; \boldsymbol{D}, 10 \mu \mathrm{m}$. 
visible along the leading process shaft (Fig. 1A, Movie 1), but protrusions collapse more frequently, resulting in overall fewer stable branches. We found no difference in branch initiation between control- and SDF1-treated interneurons making an average of two protrusions per hour (stable protrusions lasting a minimum 4 min were counted). To measure SDF1's effect on branch growth and stability, we quantified the lifetime of each protrusion. SDF1 significantly reduces the median protrusion lifetime from $36 \mathrm{~min}$ (control) to $24 \mathrm{~min}$ (SDF1; control mean = $60.9 \mathrm{~min}, \pm 2.1 \mathrm{SEM}$; SDF1 mean $=46.6 \mathrm{~min}, \pm 1.7 \mathrm{SEM}$; Fig. $1 B)$. Graphing this protrusion lifetime data as a histogram allows us to see SDF1's effects on short-lived protrusions and long-lived branches (Fig. 1B). SDF1 creates significantly more short-lived protrusions and fewer long-lived branches. These data indicate that SDF1 reduces interneuron branching not by regulating branch initiation but by reducing branch lifetime and therefore must affect branch growth and/or stabilization.

Branch growth is presumably driven by actin protrusion and supported by microtubule invasion into the growing protrusion ultimately stabilizing the branch. Because SDF1 reduces interneuron branching, we hypothesized that SDF1 treatment would result in visible changes in actin and/or microtubules. We labeled actin and microtubules in control- and SDF1-treated interneurons with fluorescent phalloidin (filamentous actin) and $\alpha$-tubulin antibodies, respectively (Fig. 1C). F-actin is concentrated at the leading process tip in control-treated interneurons, frequently in a filamentous, wedge-shaped array oriented in the direction of migration. Microtubules are present around the nucleus in the soma, throughout the leading process, and extending out into the actin-rich leading process tips. In SDF1-treated interneurons, F-actin at the leading process tip appears consolidated into a more narrow, streamlined wedge shape. F-actin is also frequently present along the leading process shaft in small protrusions (Fig. 1C, SDF1). Microtubules also appear condensed within a thinner leading process shaft but still extend out into the actin present at the leading process tips. These observations suggest that SDF1 reduces branching by regulating the cytoskeletal arrays of actin and microtubules within the leading process.

\section{Depolymerizing actin and/or microtubules changes interneuron leading process dynamics}

Many cytoskeletal regulators increase or decrease the rates of polymerization and/or change the structure of cytoskeletal arrays. We expect that if SDF1 reduces branching by regulating cytoskeletal dynamics, we should be able to phenocopy SDF1's effects on interneuron branching through application of actin and microtubule depolymerizing drugs. To test this hypothesis we first determined the consequences of blocking branch initiation and growth by inhibiting actin protrusion through treatment with Latrunculin B (LatrB), an actin depolymerizing toxin. Control interneurons maintain a dynamic leading process that branches frequently, allowing direction change (Fig. 2A, control, Movie 2). The leading process tip is wedge shaped and frequently splits to create new interneuron branches. Interneurons treated with low doses of LatrB ( $5 \mathrm{nM}, 15.8 \mathrm{~nm}$ ) are still able to maintain migratory morphology, but both branching and migration speed are reduced significantly (Fig. $2 A$, LatrB, $B, C$ ). LatrB (5 nm) causes a $52 \%$ reduction in total branching, while $15.8 \mathrm{~nm}$ LatrB causes a $79 \%$ reduction in total branching. SDF1 reduces both leading process splitting and interstitial branching, but has a greater effect on interstitial branching (Lysko et al., 2011). Thus, we analyzed LatrB's effects on both types of branching and found

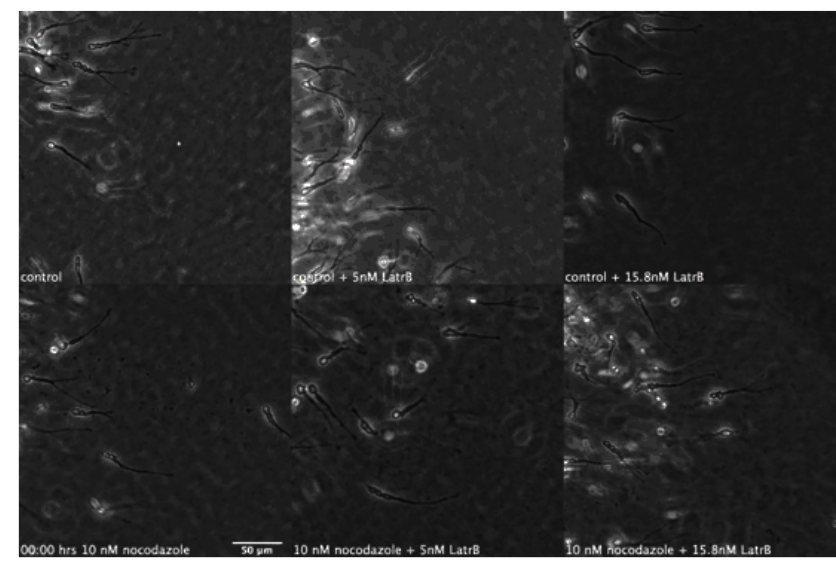

Movie 2. Depolymerizing actin and/or microtubules in migrating interneurons generates distinct migratory behaviors and morphologies. Control, Migrating interneurons display normal branching behavior, with wedge-shaped leading process tips. Control + LatrB, Depolymerizing actin with LatrB reduces interneuron branching and speed in a dose-dependent manner. Both leading process splitting and interstitial branching initiation and growth are inhibited. The tip of the leading process collapses to form a circular tip that rapidly extends and retracts. Nocodazole, Low-dose nocodazole $(10 \mathrm{~nm})$ reduces interneuron branching and speed, created a spiky, brushy leading process appearance. Nocodazole + LatrB, Combined application of LatrB and nocodazole results in further reduction of branching and speed, while reducing the brushy appearance induced by nocodazole treatment alone. $10 \times$ time-lapse phase microscopy, 1 frame/10 min. Scale bar, $50 \mu \mathrm{m}$.

that both types are similarly reduced. LatrB ( $5 \mathrm{~nm})$ reduces both types by $52 \%$, while at $15.8 \mathrm{~nm}$ leading process splitting is reduced by $83 \%$ and interstitial branching only slightly less at $73 \%$, together indicating that depolymerizing actin reduces both branching types similarly.

In interneurons treated with LatrB, the leading process tip wedge frequently collapses to a small circular tip that cycles between rapid extension and collapse without forming stable branches (Movie 2). This collapsed leading process tip contains a small patch of consolidated F-actin (Fig. 2D), in contrast to the streamlined actin wedge seen in interneurons treated with SDF1 (Fig. 1C). LatrB treatment also results in leading process elongation, narrowing the microtubules within the leading process shaft.

Next we examined the consequences of blocking microtubule invasion by treating with nocodazole, a microtubule depolymerizing drug. At high concentrations $(1 \mu \mathrm{M})$, multihour nocodazole treatment is reported to induce a loss of bipolar morphology and an increase in branching in interneurons, while acute treatment (10 min to $1 \mathrm{~h}$ ) induces nuclear movement in SVZa neurons (Schaar and McConnell, 2005; Baudoin et al., 2008). However, at low concentrations ( $20 \mathrm{~nm}$ ) of nocodazole, interneurons maintain bipolar morphology and leading process branching is reduced (Baudoin et al., 2008). We chose a similarly low dose of nocodazole $(10 \mathrm{~nm})$, to inhibit microtubule polymerization without causing complete microtubule collapse, resulting in branching reduction while maintaining interneuron migratory morphology. Low-dose nocodazole (10 nM) treatment alone results in a significant decrease in branching and a modest decrease in speed (Fig. $2 A-C$ ). While total branching is reduced by $38 \%$, leading process splitting is reduced by $37 \%$ and interstitial branching is reduced by $40 \%$. The leading process is frequently shorter with splayed microtubules and a more elaborate, brushy actin network (Fig. 2D).

Blocking microtubule polymerization while depolymerizing actin results in an additive effect; nocodazole causes a further 
decrease in branching in combination with LatrB (Fig. 2B). Interestingly, nocodazole does not cause an additive reduction in migration speed, suggesting that the balance between actin and microtubule dynamics may be more critical for efficient nucleokinesis than the absolute polymerization rates (Fig. 2 C). This combined treatment results in consolidated actin at the leading process tip but does not share the shortened leading process phenotype of interneurons treated with nocodazole only. To compare SDF1's effects on leading process length to that induced by LatrB or nocodazole, we measured the length of live-imaged interneurons. While LatrB lengthens the leading process and nocodazole treatment shortens the leading process (LatrB $=83$ $\mu \mathrm{m} \pm 20 \mu \mathrm{m} \mathrm{SD}$, nocodazole $=52 \mu \mathrm{m} \pm$ $12 \mu \mathrm{m} \mathrm{SD}, n \geq 50$ for each condition, $t$ test comparing each to control, $p<$ 0.001), SDF1 treatment does not change leading process length (control $=63$ $\mu \mathrm{m} \pm 12 \mu \mathrm{m} \mathrm{SD}, \mathrm{SDF} 1=63 \mu \mathrm{m} \pm 14$ $\mu \mathrm{m} \mathrm{SD}, n=50$ for each condition, $t$ test, $p>0.9)$. Depolymerizing actin with LatrB produces a consolidated actin phenotype similar to SDF1 treatment, while nocodazole treatment and SDF1 seem to have opposite effects on the microtubule cytoskeleton and leading process appearance. Together, these results suggest that SDF1 may decrease branching by using actin depolymerization, but also that actin depolymerization alone is not enough to phenocopy SDF1's effects on interneuron morphology.

\section{SDF1 increases calpain protease activity in migrating interneurons}

Because SDF1 reduces branching and consolidates actin at the leading process tip, we sought to identify downstream mediators that regulate actin to affect neuronal branching. Calpain protease regulates both cell adhesion and the actin network by cleaving several different substrates involved in adhesions and actin regulation (Franco et al., 2004; Franco and Huttenlocher, 2005). In hippocampal neurons, actin polymerization is repressed by calpain protease to reduce branching (Mingorance-Le Meur and O'Connor, 2009). In these neurons, exposure to branching factors such as NT3 or brain-derived neurotrophic factor (BDNF) cause cAMP levels to rise, stimulating PKA to inhibit calpain, and allow more branches to form. We have previously shown that increasing cAMP in interneurons also increases interneuron branching (Lysko et al., 2011). Conversely, we have determined that SDF1 acts via its G-coupled protein receptor CXCR4 using inhibitory $\alpha$-protein $\mathrm{G}_{\mathrm{i}}$, which typically reduces cAMP levels, to regulate interneuron migration (Lysko et
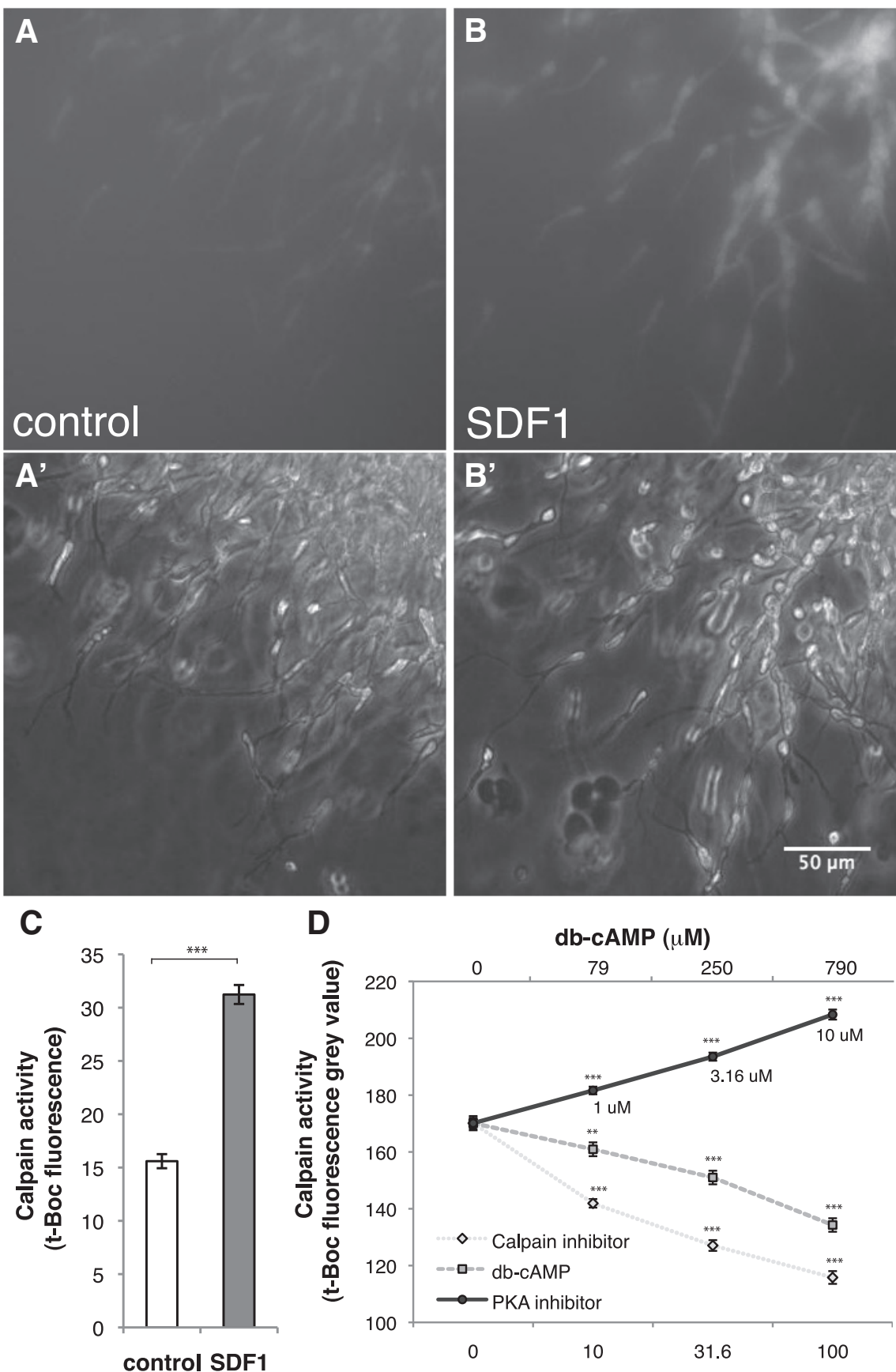

D

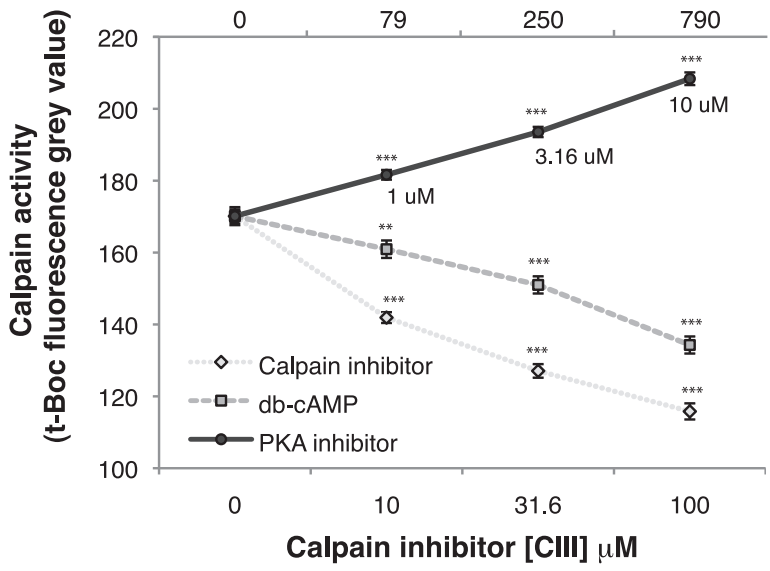

Figure 3. SDF1 increases calpain protease activity. $A, B$, Live fluorescence microscopy of migrating interneurons loaded with calpain activity indicator t-Boc, treated with control or SDF1. SDF1-treated interneurons are brighter, indicating higher calpain activity. $\boldsymbol{A}^{\prime}, \boldsymbol{B}^{\prime}$, Phase microscopy images corresponding to $\boldsymbol{A}$ and $\boldsymbol{B}$. C, Quantification of calpain activity shows that SDF1 significantly increases calpain activity ( \pm SEM; ${ }^{* * *} p<0.001, t$ test). $\boldsymbol{D}$, Inhibiting PKA using specific inhibitor PKI similarly increases calpain activity $\left({ }^{* *} p<0.001, t\right.$ test). Conversely, increasing PKA activity using PKA agonist dbcAMP reduces calpain activity, similar to reducing calpain activity directly with calpain inhibitor CIII ( \pm SEM; ${ }^{* * *} p<0.001,{ }^{* *} p<0.01, t$ test; $n \geq 345$ cells for each condition, four independent experiments); $t$ tests are treatment compared with control.

al., 2011). Based on these data we postulate that the SDF1induced reduction of interneuron branching is a consequence of increased calpain activity.

To measure calpain activity, we used the cell-permeable reporter t-Boc, a diffusible peptide substrate that fluoresces upon calpain cleavage, allowing an optical measurement of average calpain activity within the cell (Fig. $3 A$ ). SDF1 treatment significantly increased calpain activity (Fig. $3 B, C$ ). Similarly, treatment 

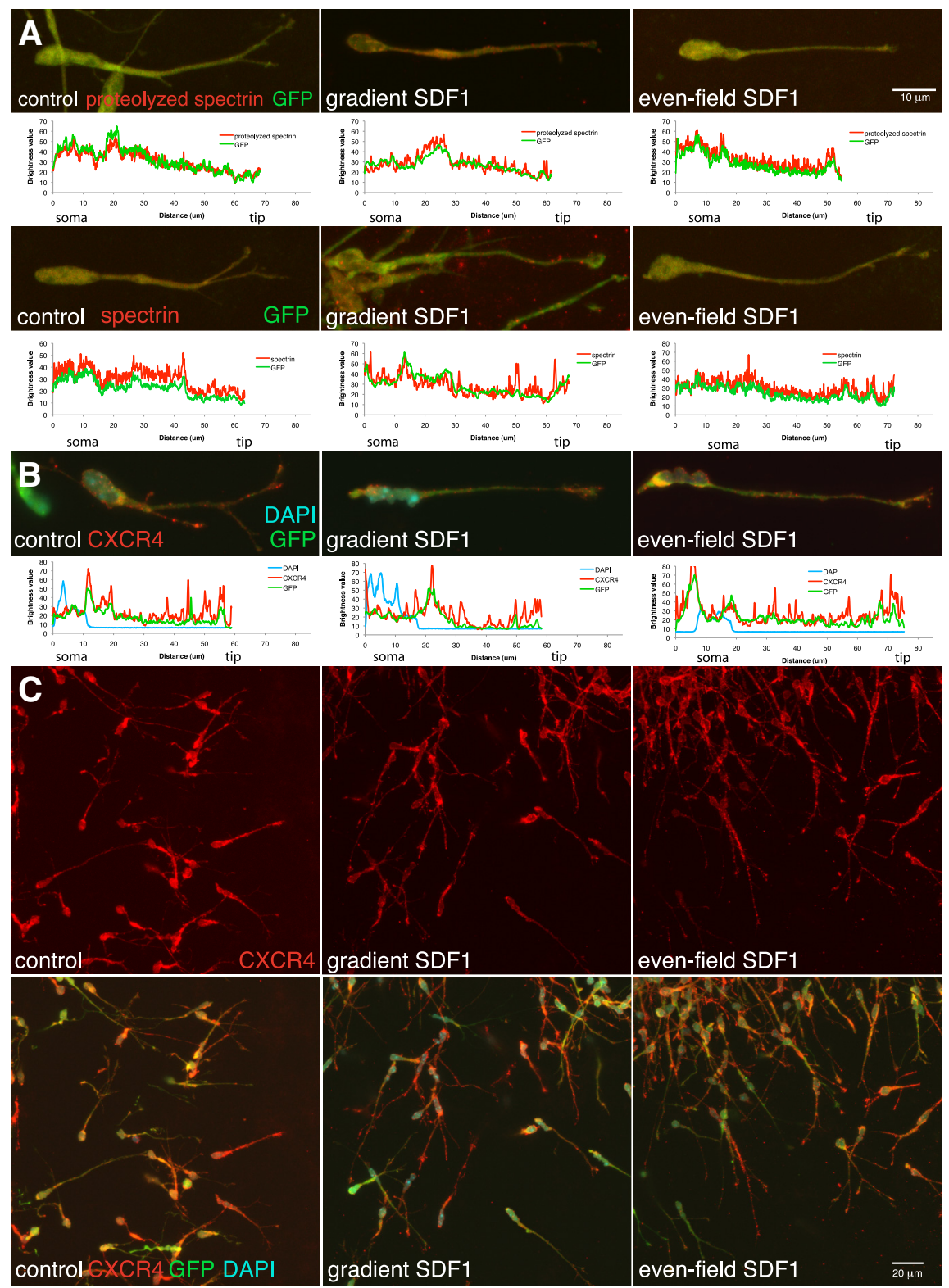

D
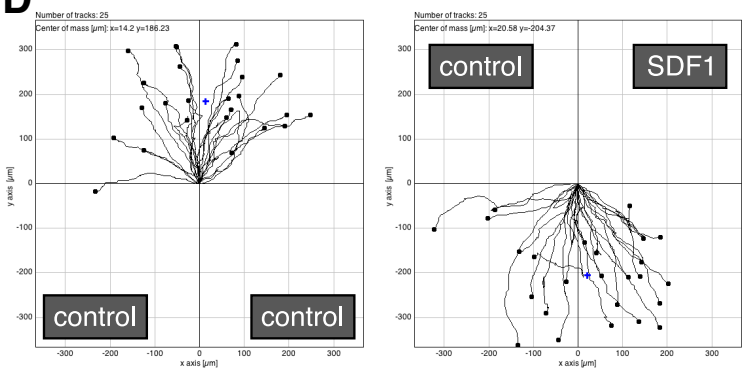

with PKI, a PKA inhibitor, also increased calpain activity (Fig. 3D). Conversely, stimulating PKA with dbcAMP decreased calpain activity, similar to the decrease seen when inhibiting calpain directly with CIII (Fig. 3D) or ALLM (data not shown), specific calpain inhibitors. This evidence shows that SDF1 treatment or inhibition of PKA increases calpain activity, while increasing cAMP reduces calpain activity.

\section{SDF1 does not regulate the polarity of} calpain activity in interneurons

Using t-Boc to measure calpain activity does not define the subcellular location of calpain activity. Calpain activity can be localized to specific areas within a neuronal process as in hippocampal neurons developing in culture (Mingorance-Le Meur and O'Connor, 2009). In these neurons, calpain activity is restricted to the soma and neurite shaft, but not present at the actin-rich growth cone, leading to a model in which calpain downregulates collateral branching at the neurite shaft. This calpain activity has been visualized by immunolabeling for proteolyzed spectrin, another substrate of calpain. To determine whether calpain activity is polarized in migrating interneurons we immunolabeled interneurons for proteolyzed spectrin and total spectrin. We used interneurons expressing cytosolic GFP to mark the cytoplasm and quantified the distribution of spectrin and GFP along the interneuron leading process. In contrast to cultured hippocampal neurons, we find that both spectrin and proteolyzed spectrin are distributed along the length of the leading process in a similar pattern as our cytoplasmic marker GFP (Fig. 4A). SDF1 treatment does not induce significant changes in the distribution of spectrin. Given that interneurons migrate through an SDF1 gradient within the neocortex in vivo, we also treated interneurons with a gradient of SDF1 to test whether a polarized source of SDF1 would induce a polarized gradient of calpain activity within interneurons. An SDF1 gradient was established using $\mu$-slide chemotaxis 3D chambers (Ibidi) and functionally tested by measuring T-cell chemotaxis (see Materials and Methods). Interneurons migrating within a gradient of SDF1 did not display polarized spectrin or proteolyzed spectrin (Fig. 4A).

Although no evidence was found that SDF1 regulates calpain activity polarity, it is possible that an SDF1 gradient could affect the distribution of the SDF1 receptor CXCR4, polarizing downstream signaling. Previous data using MGE explant 
culture has shown that CXCR4 is localized throughout the leading process and soma, including the perinuclear region, while the SDF1 receptor CXCR7 is primarily perinuclear, but present in relatively small amounts throughout the leading process and soma (Sánchez-Alcañiz et al., 2011; Wang et al., 2011). We immunolabeled interneurons in control, SDF1-gradient and SDF1-even-field conditions for CXCR4 and compared its distribution with a cytoplasmic marker, GFP. In all treatments, CXCR4 immunolabeling was observed throughout the leading process and soma and in general, was not altered by gradient or even-field SDF1. CXCR4 immunolabeling appeared punctate and small patches of puncta were frequently observed ( $>90 \%$ of interneurons in all treatments). CXCR4 immunolabeling showed a varied intensity from cell to cell that was consistent across all conditions (Fig. $4 B, C)$.

To determine whether CXCR4 distribution was polarized, we compared the relative enrichment of CXCR4 to the cytoplasmic marker GFP in three regions of the leading process: at the leading process tip, in the distal leading process, and in the process/cytoplasmic bulge proximal to the soma (Fig. 4B). At the leading process tip, enriched CXCR4 was observed in 57\% of control interneurons while in $42 \%$ CXCR4 distribution was not polarized (CXCR4 was very infrequently reduced at the leading process tip, $\sim 1 \%$; Fig. $4 B$ ). Treatment with a gradient or even-field SDF1 did not alter the polarization of CXCR4 at the tip (gradient SDF1, 56\% polarized; even-field SDF1, 58\% polarized). The amount of CXCR4 enrichment in the polarized areas was not significantly altered (average length of CXCR4 polarization: control, $4.6 \mu \mathrm{m}$; gradient SDF1, $5.1 \mu \mathrm{m}$; even-field SDF1, $4.2 \mu \mathrm{m})$. Interneuron position within different areas of an SDF1 gradient did not correlate with particular levels of CXCR4 expression or leading process tip polarization (Fig. 4C).

In the distal leading process, CXCR4 was usually present but not enriched, although in many interneurons we did observe CXCR4 enrichment $(27 \%$ of both control and gradient SDF1-treated interneurons; $41 \%$ of even-field SDF1treated interneurons; Fig. 4B). At the cytoplasmic bulge and leading process proximal to the soma, CXCR4 was usually present but not enriched (all treatments, $\sim 70 \%$ ), enriched in many interneurons (control, 23\%; gradient SDF1, 21\%; evenfield SDF1, 26\%), and reduced in the remaining interneurons. To the rear of the
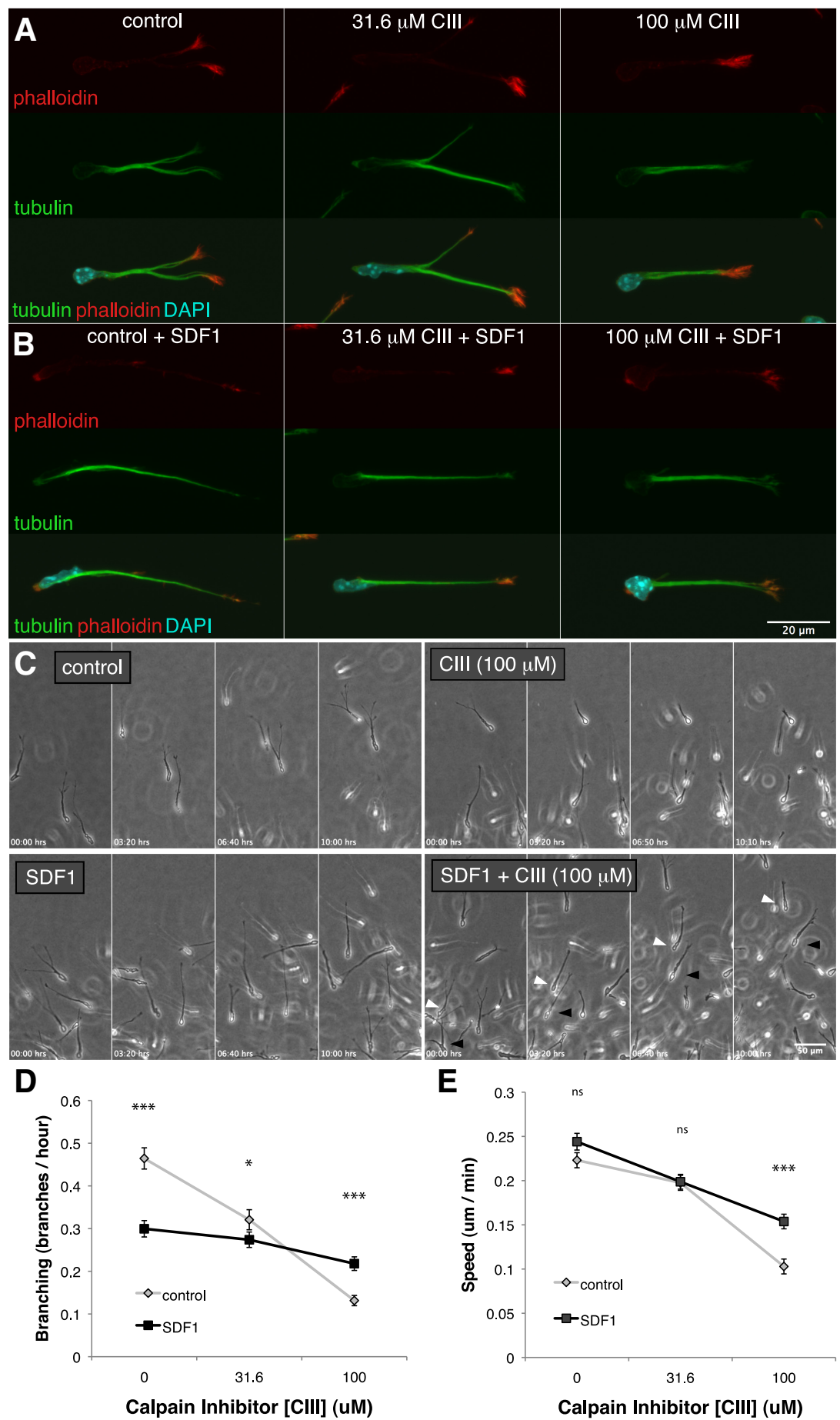

Figure 5. SDF1 regulates the actin network at the interneuron leading process tip through calpain activity. $\boldsymbol{A}$, Interneurons treated with increasing amounts of calpain inhibitor (CIII) show increased actin localization at the leading process tip. Interneurons are labeled for F-actin in red (phalloidin), microtubules in green ( $\alpha$-tubulin), and nuclei in blue (DAPI)). $\boldsymbol{B}$, Interneurons treated with SDF1 have reduced actin at the leading process tip. Calpain inhibition still increases actin localization at the leading process tip, but SDF1 dampens this effect. $C$, Frames from the time-lapse sequence of Movie 3 showing leading process dynamics during calpain inhibition alone and in combination with SDF1. Calpain inhibition reduces branching and migration, shortens the leading process, and results in a brushy leading process tip. SDF1 alone reduces branching frequency. SDF1 in combination with CIII reduces the effects of calpain inhibition, allowing more normal branching and migration. Arrowheads mark two different representative cells. $\boldsymbol{D}$, Calpain inhibition results in a dose-dependent reduction in branching. SDF1 reduces branching under control conditions, but opposes calpain inhibition to maintain branching ( $\pm \mathrm{SEM} ; p<0.001$, analysis of deviance, ${ }^{* * *} p<0.001,{ }^{*} p<0.05$, post hoc tests). $E$, Calpain inhibition decreases migration speed; however, SDF1 can increase migration speed in the presence of calpain inhibition. ( $p=0.1$ for control vs SDF1 at $0 \mu \mathrm{M}$ CIII). Post hoc and $t$ tests are SDF1-treated compared with control ( $n=75$ cells for each condition, 3 independent experiments). Scale bars: $A, B, 20 \mu \mathrm{m} ; \boldsymbol{C}, 50 \mu \mathrm{m}$. 
soma CXCR4 was enriched in some interneurons (control, 36\%; gradient and even-field SDF1, 38\%).

Together these data suggest that CXCR4 localization in migrating interneurons is nonuniform and can be polarized, but is quite varied both within different sections of the leading process and from interneuron to interneuron. This variation may be consistent with changing localization during the dynamic process of migration and this static analysis will not reveal dynamic changes in CXCR4 polarization during an interneurons' exposure to SDF1. In future studies, experiments focusing on the dynamic localization of CXCR4 will be required to elucidate the polarity of SDF1 signaling, but based on our data, treatment with gradient or even-field SDF1 does not cause large changes in CXCR4 distribution, consistent with nonpolarized SDF1 regulation of calpain activity.

We also used this opportunity to test whether the migration path of interneurons is affected by polarized SDF1. We acquired time-lapse movies and tracked the path of interneurons migrating perpendicular to the SDF1 gradient. If SDF1 can act as an attractant for interneurons in this system we hypothesized that their migration paths would deflect toward higher concentrations of SDF1. In control conditions, interneurons migrate out from an explant following a relatively straight migration path. In both even-field and gradient SDF1 conditions, interneurons also migrate relatively straight and do not deflect toward higher SDF1 concentrations (Fig. 4D; four independent experiments with SDF1-expressing HEK293T cells, at least 90 interneurons tracked per condition; two independent experiments with recombinant SDF1, 50 interneurons tracked per condition and similar to cellgenerated SDF1 gradients, data not shown). We do not observe explant shape distortion as seen when explants have been cultured near a source of a known interneuron attractant such as Nrg1 (Flames et al., 2004). Although interneurons concentrate near SDF1-coated beads implanted in brain slices (Li et al., 2008), in this in vitro system, interneurons do not appear to migrate toward SDF1, consistent with the observation that interneurons migrate down the concentration gradient of SDF1 as they migrate dorsally through the SVZ of the neocortex (Stumm et al., 2007; Li et al., 2008; López-Bendito et al., 2008). Together these results indicate that SDF1 signaling does not change the distribution of CXCR4 and does not regulate calpain activity in a polarized manner, but increases calpain activity evenly throughout the leading process to affect branching within the entire leading process.

\section{Calpain activity regulates actin protrusion at the leading process tip}

If SDF1 increases calpain activity to depolymerize actin and reduce branching, we hypothesized that calpain inhibition might increase actin polymerization. Interneurons treated with calpain inhibitor $(31.6 \mu \mathrm{M}, 100 \mu \mathrm{M})$ were stained with fluorescently labeled phalloidin to visualize actin polymerization. While the tip of the interneuron leading process is normally enriched with F-actin, inhibiting calpain results in an expansion of actin at the leading process tip (Fig. 5A). To determine whether calpain inhibition increases leading process tip area, actin within the leading process tip, or both, interneurons expressing GFP were treated with calpain inhibitor, fixed and stained for phalloidin and GFP, and actin positive area and leading process tip area were measured. In virtually all interneurons examined ( $>99 \%, n=200)$, including both control and calpain-inhibited interneurons, F-actin is present throughout the leading process tip area (the leading process tip area is equal to the actin positive area at the leading process tip). In control interneurons the average actin

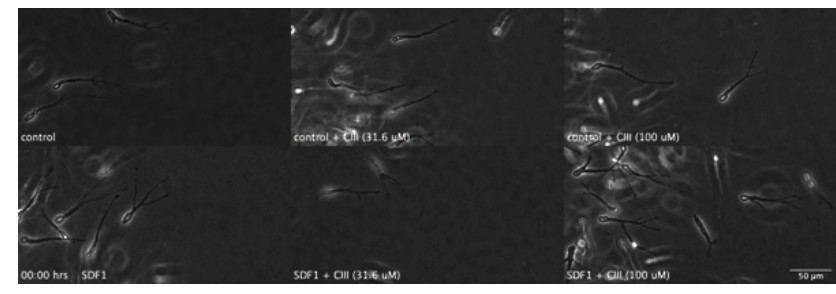

Movie 3. SDF1 regulates leading process tip protrusion by activating calpain. Control, Migrating interneurons display normal branching behavior. Control + CIII, Calpain inhibition, using specific inhibitor CIII, reduces interneuron branching and speed while inducing a broader, more protrusive leading process tip. The length of the leading process is also reduced. SDF1, SDF1 treatment alone reduces branching and increases speed. SDF1 + CIII, In combination with calpain inhibitor CIII, SDF1 partially prevents calpain inhibition's effects, resulting in more normal branching, speed, and leading process tip phenotype. $10 \times$ time-lapse phase microscopy, 1 frame/10 min. Scale bar, $50 \mu \mathrm{m}$.

positive area per cell is $20 \mu \mathrm{m}^{2} \pm 8 \mu \mathrm{m}^{2}$ SD. Calpain inhibition significantly increased the actin positive area per cell $(31.6 \mu \mathrm{M}$ $\mathrm{CIII}=29 \mu \mathrm{m}^{2} \pm 11.8 \mu \mathrm{m}^{2} \mathrm{SD} ; 100 \mu \mathrm{M} \mathrm{CIII}=32 \mu \mathrm{m}^{2} \pm 11.4$ $\mu \mathrm{m}^{2} \mathrm{SD} ; n=165$ cells for each condition, three independent experiments, $t$ tests compared with control, $p<0.001)$. With increasing calpain inhibition, the actin positive area within the cell's leading process is concentrated in only one leading process tip (actin positive area per leading process tip, control $=13 \mu \mathrm{m}^{2}$, $31.6 \mu \mathrm{M}$ CIII $=21 \mu \mathrm{m}^{2}, 100 \mu \mathrm{M}$ CIII $=27 \mu \mathrm{m}^{2}$ ). In interneurons treated with SDF1 alone, actin is reduced within the leading process tip as seen previously (Fig. 1). Inhibiting calpain in the presence of SDF1 also expands F-actin at the leading process tip, but this affect is dampened by SDF1 (Fig. 5B).

Because calpain inhibition expands the actin positive area at the interneuron leading process tip, we were interested in examining the effect of calpain inhibition on interneuron leading process dynamics and migration. Recognizing that calpain regulates many different proteins involved in cell adhesion in addition to actin protrusion (Wells et al., 2005), it seemed reasonable to expect a complex phenotype. We analyzed the leading process branching and migration of calpain-inhibited interneurons (Fig. $5 C$; Movie 3). Calpain inhibition caused striking changes in leading process dynamics and decreased both branching and speed (Fig. 5D,E). Calpain-inhibited interneurons have an extremely dynamic leading process tip that is wider and highly protrusive, similar to a migrating fibroblast's lamellipodia. Together our data are consistent with calpain inhibition increasing actin protrusion to the extent that normal branches cannot form. We have previously noted that SDF1 treatment dampened the effects of inhibiting calpain, resulting in more normal actin at the leading process tip. We then predicted that SDF1 treatment would also dampen the deleterious effects of calpain inhibition on leading process dynamics and migration. Because SDF1 increases calpain activity (Fig. 3), we tested whether SDF1 could reverse the effects of calpain inhibition. SDF1 results in a modest increase in speed in control conditions and at the highest dose of calpain inhibition. Importantly, in interneurons treated with calpain inhibitor, SDF1 partially restores the ability to branch and migrate normally (Fig. 5C,D, Movie 3). These results indicate that SDF1 opposes calpain inhibition's effects on migration, further supporting the idea that SDF1 reduces interneuron branching by increasing calpain activity.

SDF1 increases cortactin proteolysis, reducing cortactin and consolidating actin at the leading process tip Calpain reduces cell protrusion during fibroblast migration through its cleavage of the actin regulatory protein cortactin 
(Perrin et al., 2006). Cortactin activates Arp2/3 to assist in actin nucleation and stabilizes actin branch points, promoting branched network protrusion (Weaver et al., 2001), but cortactin's role in migrating interneurons is unknown.

In cultured hippocampal neurons, actin protrusion is negatively regulated by calpain protease cleavage of cortactin, consolidating the actin network and reducing branching in areas of high calpain activity (Mingorance-Le Meur and O'Connor, 2009). In migrating interneurons, regulating branched actin network formation at the leading process tip would be especially suited to reducing leading process splitting, the more frequent mechanism of interneuron branching.

Because calpain inhibition excessively increased leading process tip protrusion, we predicted that calpain inhibition would prevent proteolysis of cortactin in treated interneurons. Increasing inhibition of calpain in migrating interneurons results in increasingly fainter proteolysis fragments on a cortactin Western blot (Fig. 6A), similar to published results using HEK293T cells (Perrin et al., 2006). Because SDF1 increases calpain activity, we predicted that SDF1 treatment would increase cortactin proteolysis. As expected, cortactin proteolysis is increased in interneurons treated with SDF1 (Fig. $6 B)$. To test whether SDF1-induced cortactin proteolysis would result in observable differences at the leading process tip, we immunolabeled interneurons for cortactin in control- and SDF1-treated cells. Cortactin is primarily localized at the tips of most leading process branches and in lower concentrations along the leading process shaft, appearing in the cytoplasmic bulge that precedes the nucleus, as well as to the rear of the nucleus in some cells (Fig. 6C). Cortactin at the tip of branches colocalizes with phalloidin labeling of F-actin where it is well positioned to regulate the actin network. To test the consequences of SDF1 signaling on cortactin's localization at the leading process tip, we quantified the cortactin immunopositive area at branch tips in control- and SDF-treated interneurons. In SDF1-treated interneurons, the cortactin positive area is reduced by $>50 \%$, suggesting that the actin network will be consolidated in interneurons migrating in SDF1-expressing areas (Fig. 6D,E).

To quantify how this loss of cortactin affects actin within leading process tips, we used fluorescent phalloidin to label F-actin in control- and SDF1-treated interneurons. In control interneurons, F-actin is highly concentrated at leading process tips and organized into a wedge shape, widest at the leading edge (Fig. 6C).

E bar, $10 \mu \mathrm{m}$.
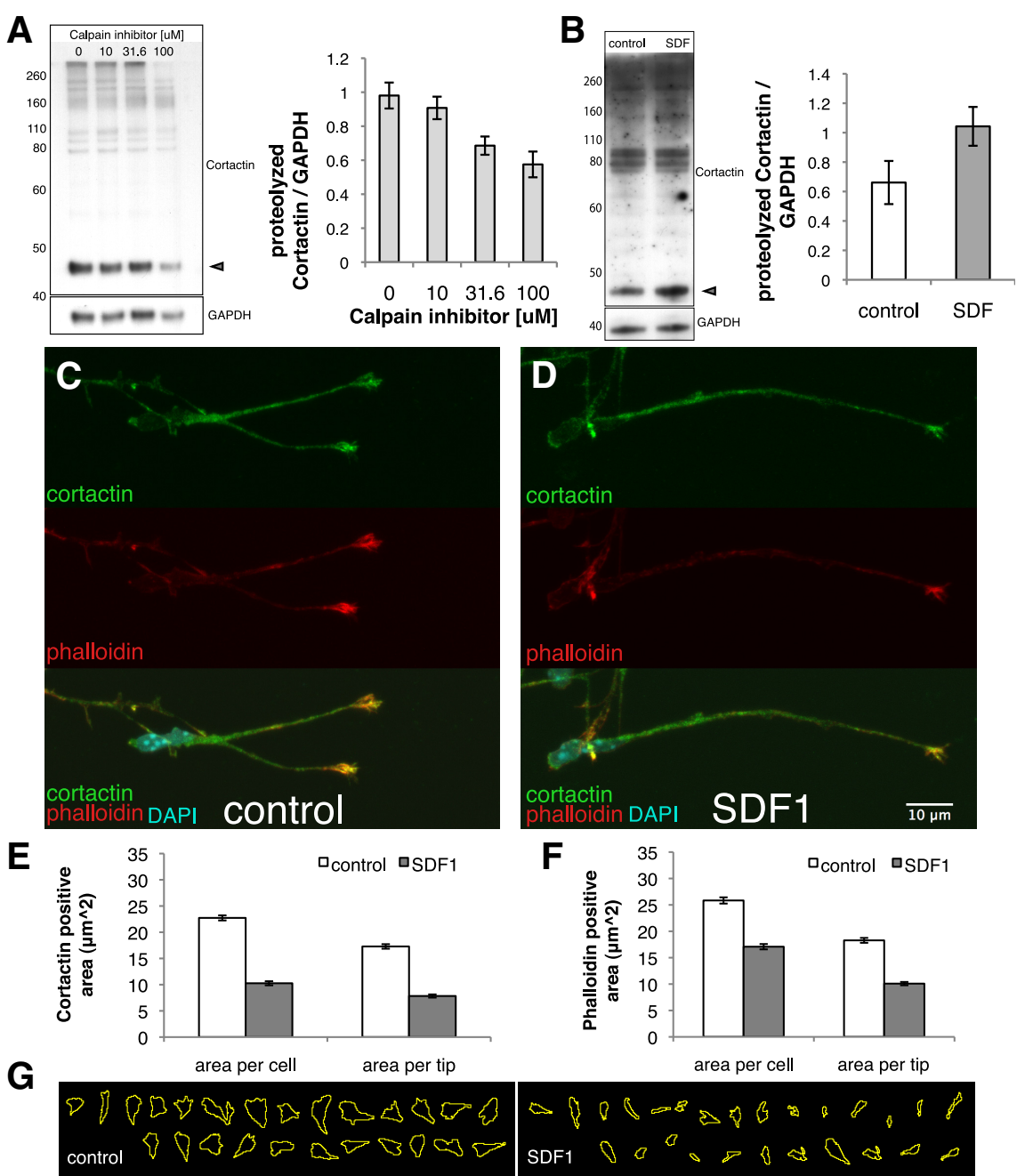

Figure 6. SDF1 increases cortactin proteolysis, reducing cortactin and consolidating actin at the leading process tip. $\boldsymbol{A}$ Migrating interneurons treated with increasing amounts of calpain inhibitor show decreasing amounts of cortactin proteolysis on an anti-cortactin Western blot. Full-length cortactin migrates at 80 and $85 \mathrm{kDa}$, while proteolyzed cortactin migrates at $\sim 46 \mathrm{kDa}$ (gray arrowhead). A small and consistent amount of cell death occurs at the highest concentration of calpain inhibitor, so proteolyzed cortactin bands were quantified and normalized to GAPDH in three independent experiments, showing a dose-dependent decrease in cortactin proteolysis. $\boldsymbol{B}$, SDF1 increases cortactin proteolysis in migrating interneurons (gray arrowhead). Proteolyzed cortactin bands were quantified and normalized to GAPDH in four independent experiments. $\boldsymbol{C}$, Cortactin immunolabeling shows that cortactin is localized throughout the leading process and is enriched at the tip of the leading process, where it colocalizes with F-actin (phalloidin labeling). D, In SDF1-treated interneurons, the cortactin positive area at the leading process tip is significantly smaller. SDF1 similarly decreases the actin positive area at the leading process tip. $\boldsymbol{E}$, Quantification of cortactin immunolabeling at the leading process tips of control- and SDF1-treated interneurons. The cortactin positive area across all branch tips within an entire cell is significantly reduced, as well as the cortactin positive area at each leading process tip ( \pm SEM; ${ }^{* *} p<0.001, t$ test). $\boldsymbol{F}$, Quantification of the actin positive area at leading process tips in control- and SDF1-treated interneurons. Similar to cortactin, the actin positive area across all branch tips within an entire cell is significantly reduced, as well as the actin positive area at each leading process tip ( \pm SEM; ${ }^{* * *} p<0.001, t$ test). $\mathbf{G}$, Outlines of actin positive areas at the leading process tip in control- and SDF1-treated interneurons. The actin positive area at the tip of the leading process is a broad wedge shape, widest at the leading edge, tapering down to the width of the leading process shaft at the rear. In the presence of SDF1, the actin positive area is consolidated and loses the pronounced wedge shape, displaying a more streamlined, rounded leading edge (300 cells for each condition for immunolabeling, 3 independent experiments). Scale

In interneurons treated with SDF1, F-actin is still present at the leading process tip, although less intensely labeled, and organization of actin appears collapsed or consolidated into a more streamlined shape (Fig. 6D). The F-actin positive area is significantly reduced by SDF1 treatment (Fig. 6F). Outlines of the F-actin positive area in control cells show the typical wedge shape at the tip of the leading process, compared with the streamlined 

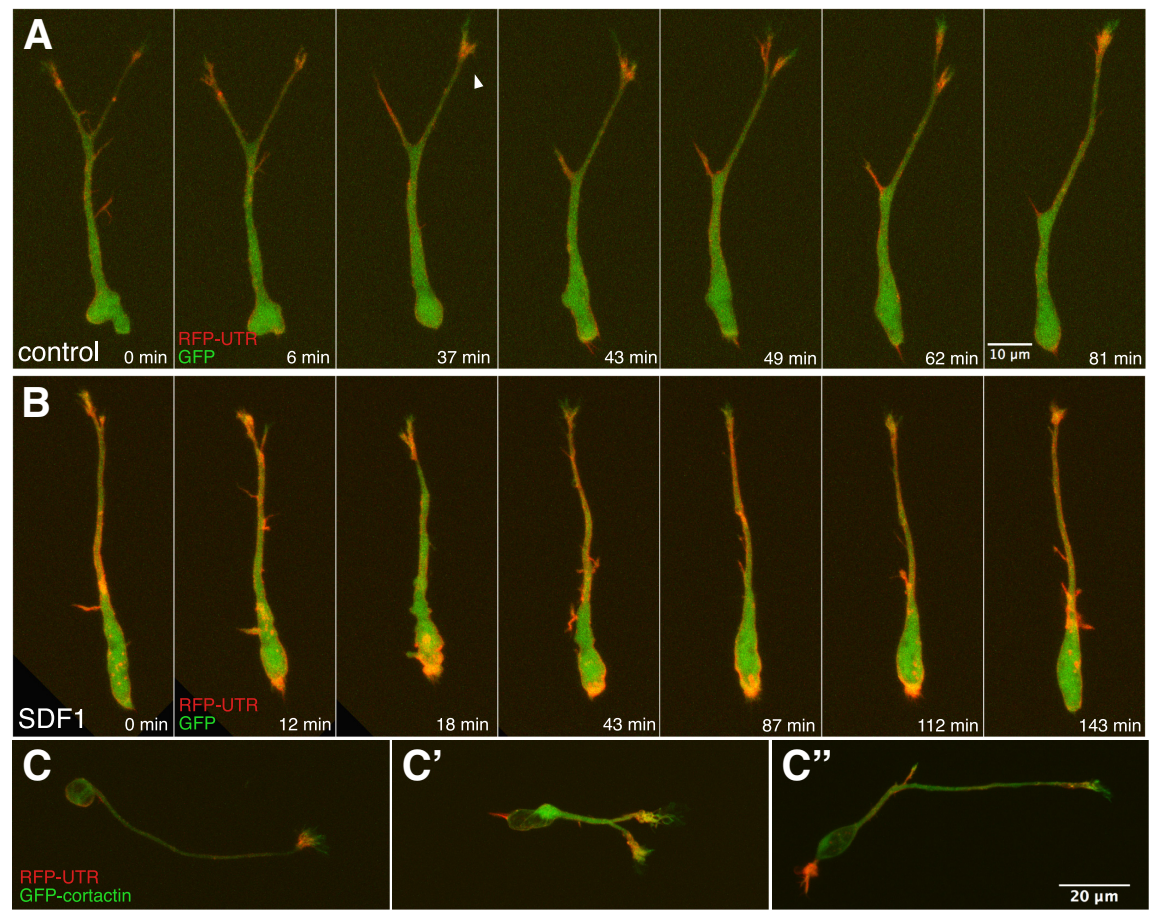

Figure 7. SDF1 changes the appearance and distribution of F-actin during interneuron migration. $A, B$, Frames from the time-lapse sequence of Movie 4 showing interneurons expressing RFP-UtrCH to label F-actin and GFP to label the cytoplasm. $A$, As a control-treated interneuron migrates, it retracts branches and undergoes a leading process split to create a new branch, while displaying the characteristic wedge shape of actin at the leading process tip (arrowhead). $\boldsymbol{B}$, In an SDF1-treated interneuron, SDF1 consolidates actin at the leading process tip, limiting branching. RFP-UtrCH signal indicates that actin is increased along the sides of the leading process shaft and is active at the rear of the soma during nucleokinesis. C, Interneurons expressing RFP-UtrCH and GFP-cortactin show that GFP-cortactin precedes and overlaps with the actin-rich wedge at the tip of the leading process. GFPcortactin and F-actin are both present at the tips of branches during leading process splitting $\left(\boldsymbol{C}^{\prime}\right)$ and interstitial (collateral) branching $\left(\boldsymbol{C}^{\prime \prime}\right)$. Scale bars: $\boldsymbol{A}, \boldsymbol{B}, 10 \mu \mathrm{m} ; \boldsymbol{C}, 20 \mu \mathrm{m}$.

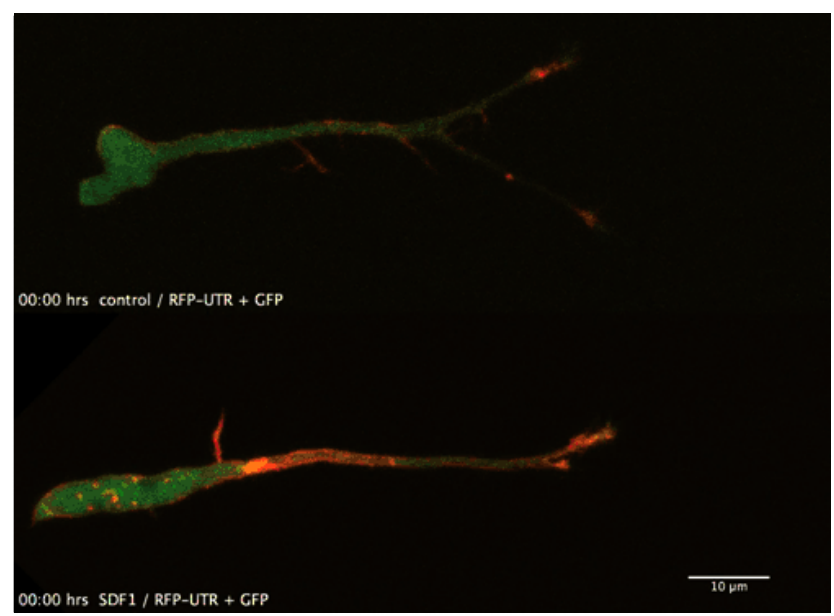

Movie 4. SDF1 changes the appearance and distribution of F-actin during interneuron migration. Interneurons expressing the F-actin probe RFP-UTR and GFP as a cytoplasmic marker. Control, top, Control interneurons generate leading process splits and interstitial branches. As branches extend actin is typically present in a wedge shape at the tip of leading process branches. Actin is also present in spiky filopodial protrusions forming along the shaft of the leading process. SDF1, bottom, Interneurons treated with SDF1 can create leading process splits, but the resulting branches are not as stable. Actin is more consolidated at the leading process tip than in control cells. Many short-lived filopodial protrusions are formed along the shaft of the leading process in SDF1-treated interneurons. Actin is present at the rear of controland SDF1-treated interneurons during nucleokinesis. $60 \times$ time-lapse confocal microscopy, 1 frame $/ 6 \mathrm{~min}$. Scale bar, $10 \mu \mathrm{m}$ shape of the tip of SDF1-treated interneurons (Fig. 6G). This streamlined shape contrasts with the consolidated actin patch seen in interneurons treated with LatrB (Fig. 2), suggesting that SDF1's regulation of actin is distinct from simple actin depolymerization, consistent with cortactin's structural role in actin networks. These data indicate that when SDF1 increases calpain proteolysis of cortactin, the branched actin network becomes consolidated and less able to support branching.

\section{SDF1 consolidates actin at the leading process tip to reduce branching}

We have shown through phalloidin labeling of fixed interneurons that SDF1 consolidates actin at the leading process tip. To examine actin localization in live interneurons and observe SDF1's effects on actin during the dynamic process of interneuron migration, we performed timelapse imaging of interneurons expressing RFP-Utrophin-CH (RFP-UtrCH) to show F-actin localization and GFP to highlight the cytoplasm. RFP-UtrCH is an F-actin probe based on the calponin homology domain of utrophin that binds F-actin and reports the normal distribution of F-actin in live cells, including migrating neurons (Burkel et al., 2007; Solecki et al., 2009). In control-treated interneurons, actin is present at leading process tips of extending and retracting branches (Fig. 7A, Movie 4). At the most distal tip of the leading process a small area of cytoplasm contains less actin. When the leading process splits to form new branches, actin is present in a wedge shape before the split (Fig. 7A, arrowhead). Small, actin-rich collateral protrusions similar to filopodia are observed along the shaft of the leading process. Actin is also observed at the periphery of the shaft along the length of the leading process. Actin is not commonly localized to the bulge proximal to the nucleus but is present in the trailing process and rear cortex of the soma during nucleokinesis.

In SDF1-treated interneurons, actin at the tip of the leading process appears more consolidated, changing from a broad wedge shape to a more streamlined shape (Fig. $7 B$, Movie 4). However, SDF1 treatment does increase the appearance of actin along the shaft of the leading process. The leading process is still capable of branching, but nascent branches frequently collapse. Small collateral protrusions are observed with similar frequency as in control interneurons (control, 3.4/h, SDF1, 3.5/h, $n=11$ cells per condition), consistent with our data that SDF1 does not inhibit branch initiation. In SDF1-treated interneurons these protrusions do not usually form stable branches, similar to the short-lived protrusions observed during phase microscopy in Figure 1. We hypothesized that SDF1 might increase the frequency of actin visible at the rear cortex of the cell soma involved in nucleokinesis, but although a small increase was observed (control, $0.8 / \mathrm{h}, \mathrm{SDF} 1,1.3 / \mathrm{h}, n=11$ ), this difference did not reach levels of significance. These time-lapse experiments confirm our observations in fixed cells that SDF1 con- 


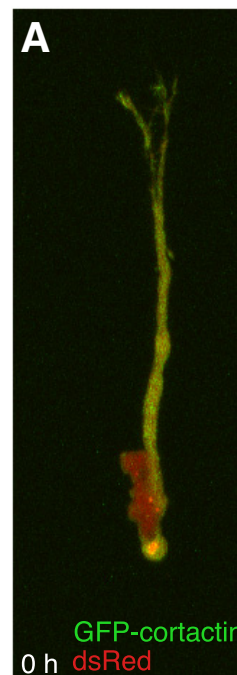

B
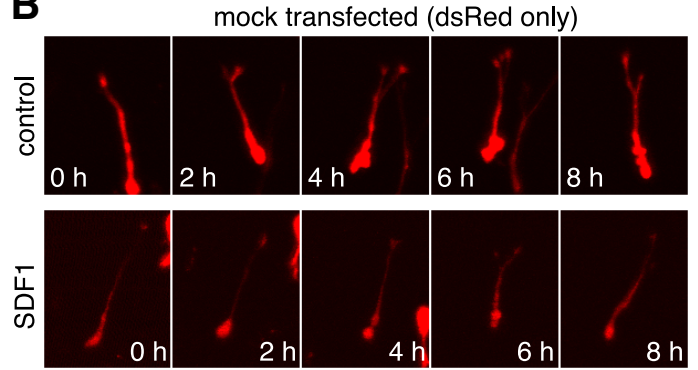

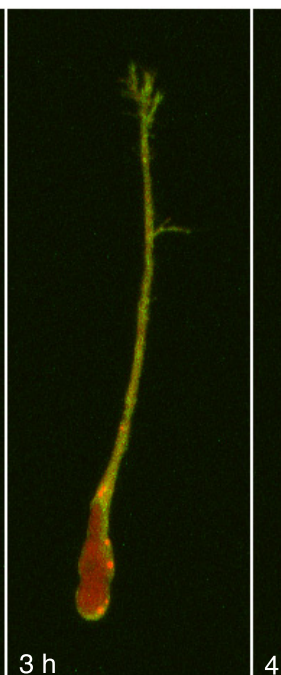

$3 \mathrm{~h}$

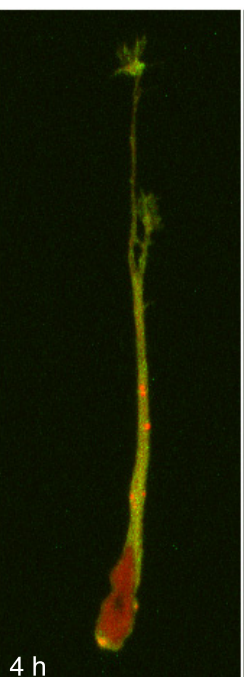

$4 \mathrm{~h}$
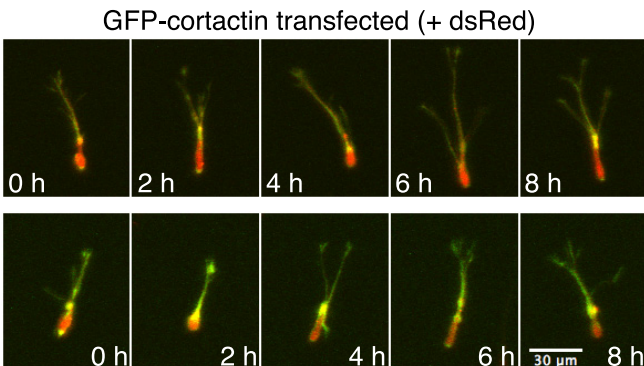
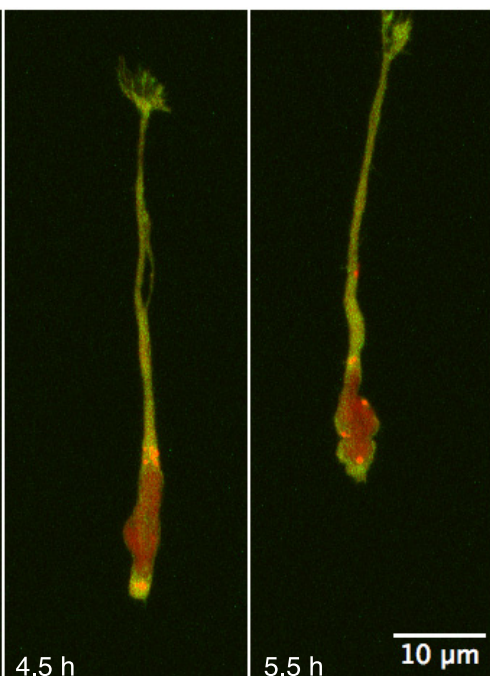

C

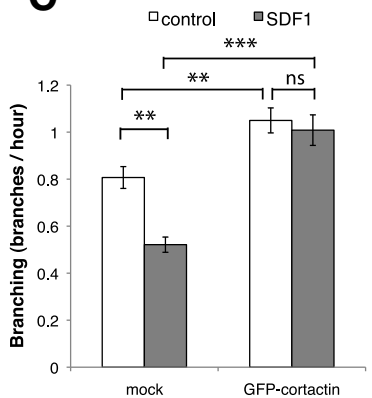

Figure 8. Cortactin remains localized to the tip of the leading process during interneuron migration and is sufficient to block SDF1 reduction of branching. $A$, Frames from the time-lapse sequence of Movie 5. Cortactin is localized throughout the leading process and enriched at leading process branch tips. B, Frames from the time-lapse sequence of Movie 6. SDF1 reduces branching compared with control-treated interneurons. Overexpressing cortactin increases branching significantly in control-treated interneurons, and blocks SDF1 reduction of branching. $C$, Quantification of branching frequency for the experiment in $\boldsymbol{B}\left( \pm\right.$ SEM; $p<0.001$, analysis of deviance, ${ }^{* * *} p<0.001,{ }^{* *} p<0.01$, post hoc tests). Scale bars: $\boldsymbol{A}, 10 \mu \mathrm{m} ; \boldsymbol{B}, 30 \mu \mathrm{m}$.

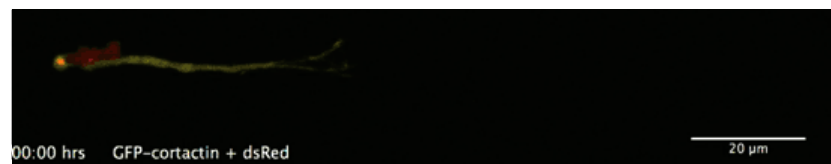

Movie 5. Cortactin is localized to the tip of the leading process. Interneuron expressing GFP-cortactin and dsRed as a cytoplasmic marker. GFP-cortactin localizes throughout the leading process and is enriched at the tip of the leading process. GFP-cortactin is localized to the tips of most growing branches. $60 \times$ time-lapse confocal microscopy, 1 frame $/ 6 \mathrm{~min}$. Scale bar, 20 $\mu \mathrm{m}$.

solidates actin at the leading process tip and reduces stable branch growth.

Our data support the hypothesis that SDF1 consolidates actin through regulation of cortactin. To confirm the colocalization of F-actin and cortactin in live interneurons we coexpressed RFPUtrCH and GFP-cortactin. F-actin and GFP-cortactin overlap primarily at the tip of the leading process in single and branched leading processes (Fig. 7C). While both localize to the tips of branches, GFP-cortactin is present at the most distal tips of both split leading processes (Fig. $7 C^{\prime}$ ) and interstitial (collateral) branches (Fig. $7 C^{\prime \prime}$ ). F-actin is localized just behind cortactin within these leading process branch tips. These results demonstrate that cortactin is positioned to regulate the growing actin network in the tips of migrating interneurons.

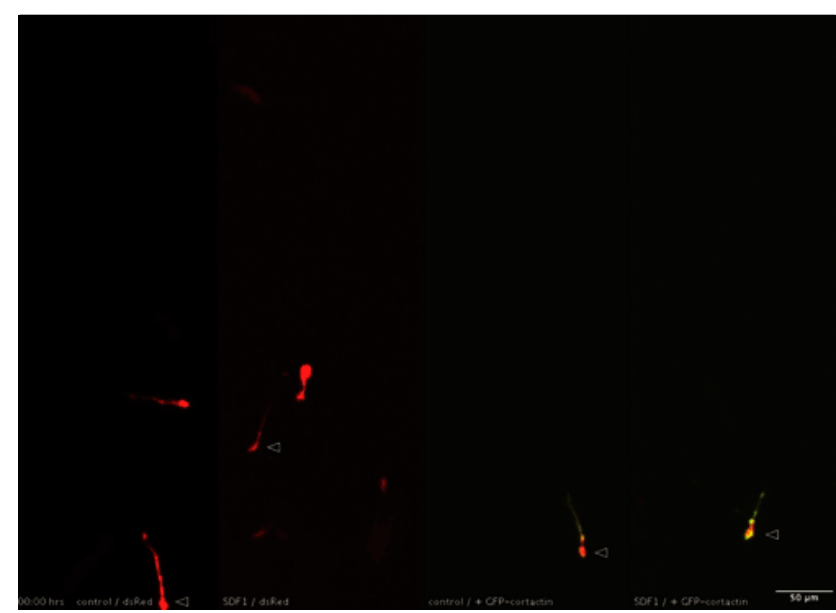

Movie 6. Overexpression of cortactin induces branching in interneurons and is sufficient to block SDF1-branching reduction. Interneurons expressing dsRed as a cytoplasmic marker and GFP-cortactin. Arrowheads mark interneurons typical for each treatment. Control/dsRed, Interneurons expressing dsRed display normal branching and migration behavior under control conditions. SDF1/dsRed, Interneurons expressing dsRed and treated with SDF1 display reduced branching. Control/+GFP-cortactin, Interneurons expressing both dsRed and GFP-cortactin display significantly more branching than control cells. SDF1/+GFP-cortactin, Interneurons expressing dsRed and GFP-cortactin and treated with SDF1 display significantly more branching than control cells, similar to GFP-cortactin-expressing cells under control conditions. SDF1 does not reduce the branching of GFP-cortactin-expressing interneurons. $20 \times$ time-lapse confocal microscopy, 1 frame $/ 10 \mathrm{~min}$. Scale bar, $50 \mu \mathrm{m}$. 


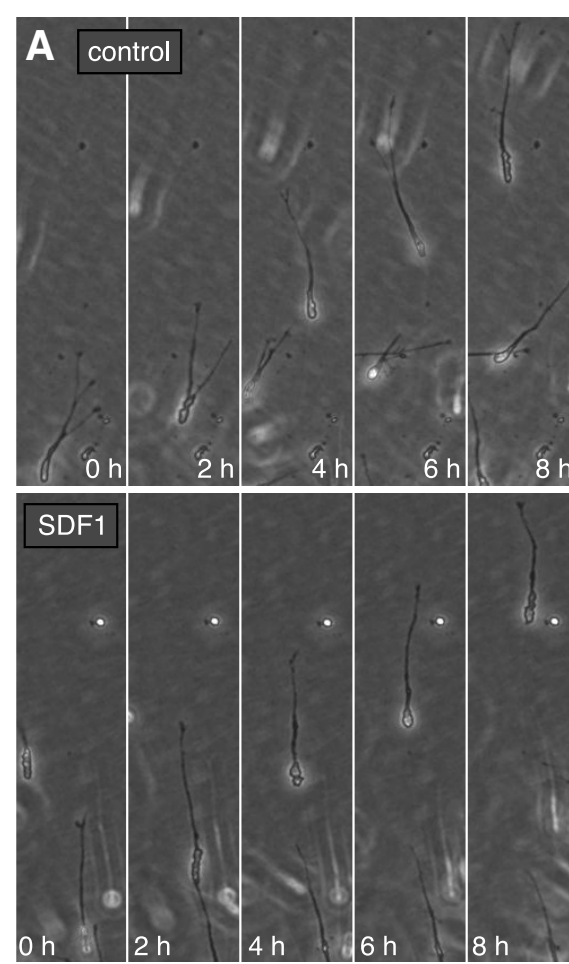

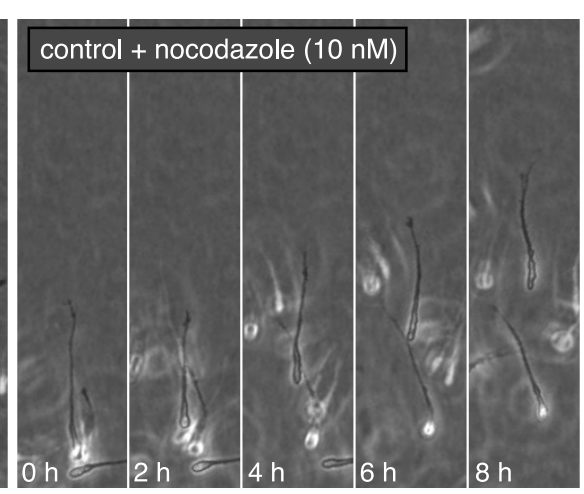

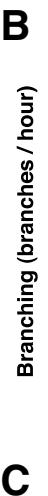

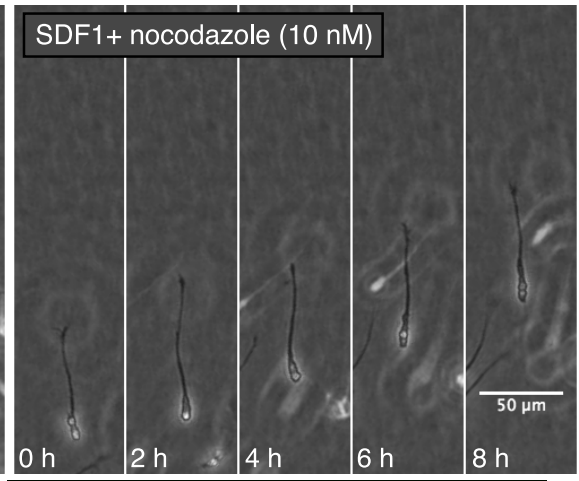

C

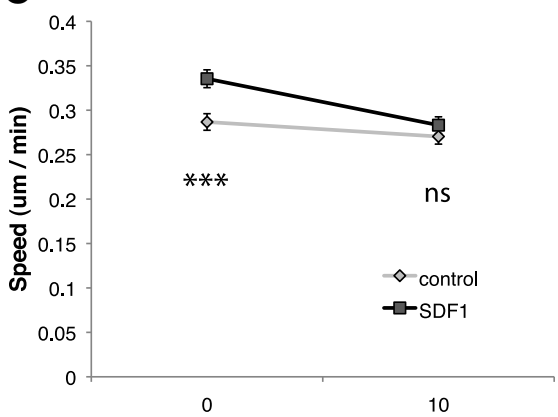

Nocodazole (nM)

E

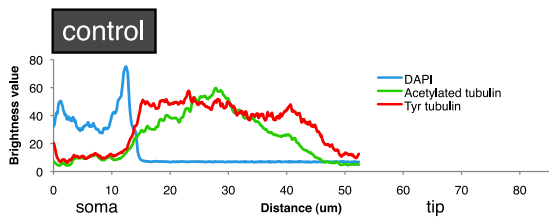

control + nocodazole $(10 \mathrm{nM})$

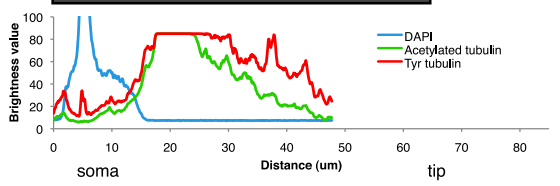

SDF1

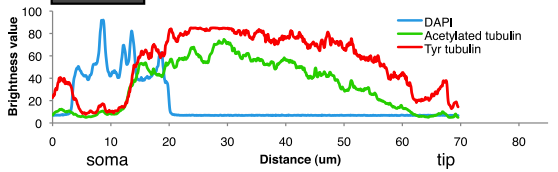

SDF1+ nocodazole (10 nM)

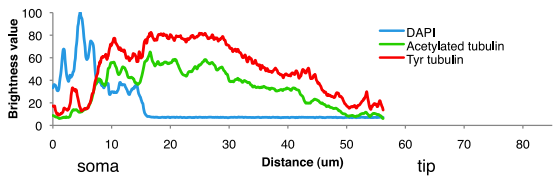

Figure 9. SDF1 stabilizes the microtubule array in the leading process. A, Frames from the time-lapse sequence of Movie 7. $\boldsymbol{B}$, Quantification of branching frequency of migrating interneurons. Treatment with $10 \mathrm{~nm}$ nocodazole reduces interneuron branching. SDF1 also reduces interneuron branching; however, treatment with nocodazole and SDF1 together does not result in additional branching reduction, indicating that SDF1 stabilizes the microtubule array and prevents additional nocodazole depolymerization of branches ( \pm SEM; $p<0.001$, analysis of deviance, ${ }^{* * *} p<0.001$, post hoc tests). C, SDF1 increases migration speed; in the presence of nocodazole, interneuron speed is inhibited under both control and SDF1 conditions ( \pm SEM; ${ }^{* * *} p=0.001, t$ test). $\boldsymbol{D}$, Immunolabeling of stable (acetylated, green) and newly formed (tyrosinated, red) microtubules. SDF1 condenses microtubules to generate an unbranched leading process, while a newly formed protrusion containing tyrosinated tubulin projects off the main leading process (white arrowhead). Low-dose nocodazole reduces branching while causing a brushy, spiky appearance at the leading process tip. In combination with SDF1, nocodazole does not additionally reduce branching, and the leading process phenotype has condensed microtubules similar to SDF1-only, in contrast to the nocodazole-only phenotype. $\boldsymbol{E}$, Quantification of the intensity of immunolabeling in $\boldsymbol{D}$. Dynamic tyrosinated tubulin is more prominent at tips of leading process branches than stable acetylated tubulin. Low-dose nocodazole or SDF1 treatment does not drastically alter the distribution of acetylated and tyrosinated tubulin. Scale bars: $\boldsymbol{A}, 50 \mu \mathrm{m} ; \boldsymbol{D}, 10 \mu \mathrm{m}$. 
Cortactin is present at the leading process tip throughout migration and the branching process

To better characterize cortactin's function throughout the process of interneuron branching, we overexpressed GFP-cortactin in migrating interneurons. GFP-cortactin appears throughout the leading process but is most concentrated at the tips of the leading process and growing branches (Fig. 8A, Movie 5). Within the tip of the leading process, GFP-cortactin can appear throughout the wedge, especially where the wedge meets the shaft of the leading process. GFP-cortactin frequently has a filamentous appearance, both at the tip and in spiky protrusions along the shaft of the leading process. GFP-cortactin does not localize to established branch points, but appears at the tip of both leading process splits and interstitial branches. This localization is consistent with cortactin's role in regulating actin at the cell periphery and confirms that cortactin is well positioned to regulate branch growth throughout the branching process.

\section{Overexpression of cortactin induces interneuron branching and is sufficient to block SDF1 reduction of branching}

Cortactin assists Arp2/3 in nucleating new actin polymers to create a branched actin network (Weaver et al., 2001). Overexpressing cortactin causes increased branching in hippocampal neurons (Mingorance-Le Meur and O'Connor, 2009), so we hypothesized that the same would occur in migrating interneurons. To quantify the effect of overexpressing cortactin on interneuron branching, we coexpressed GFP-cortactin and dsRed to label the cytoplasm of migrating interneurons. In dsRed-only control interneurons, branching occurs at a normal rate, while interneurons expressing GFPcortactin branch at a significantly higher rate (Fig. 8 B, C, Movie 6). GFP-cortactinexpressing cells are capable of maintaining multiple branches simultaneously. These results confirm that cortactin is sufficient to induce higher levels of branching in interneurons. Because we hypothesize that SDF1 downregulates cortactin to reduce branching, we predicted that overexpressing cortactin should block SDF1's reduction in branching. While SDF1 treatment of dsRed-only interneurons does reduce branching frequency, overexpressing GFP-cortactin prevents SDF1 from reducing branching (Fig. $8 B, C$, Movie 6).Together, these data support our model that SDF1 consolidates the actin network by downregulating cortactin to reduce interneuron branching.

SDF1 stabilizes the microtubule array in the distal leading process

In addition to actin-based protrusion, microtubule invasion is essential for stabilizing branches. Microtubule polymerization, stabilization, and depolymerization are affected by the binding of various MAPS that can affect both microtubule dynamics and organization (Cassimeris,

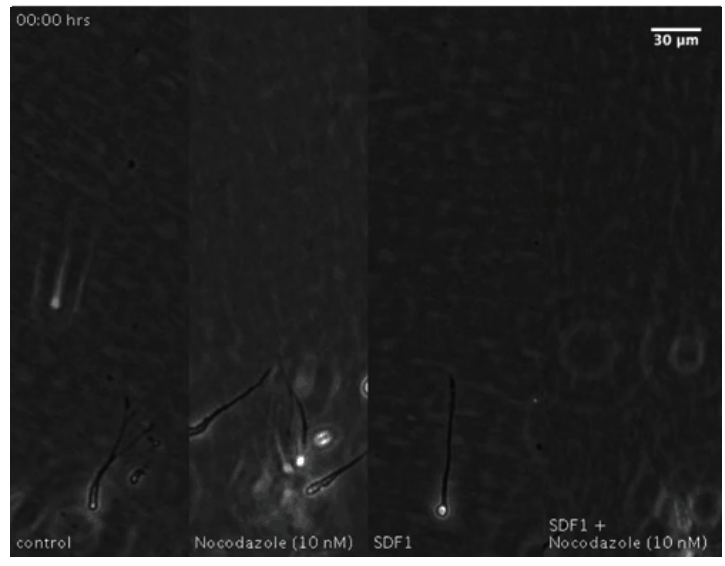

Movie 7. SDF1 stabilizes the microtubule array in the leading process. Control, Control-treated interneurons display frequent branching. Nocodazole, Nocodazole-treated interneurons have a brushy leading process appearance and reduced branching. SDF1, Interneurons treated with SDF1 have reduced branching. SDF1 + nocodazole, Interneurons treated with both nocodazole and SDF1 have reduced branching similar to SDF1 alone. Nocodazole combined with SDF1 does not collapse the leading process, indicating that SDF1 stabilizes the interneuron leading process. $10 \times$ time-lapse phase microscopy, 1 frame/10 min. Scale bar, $30 \mu \mathrm{m}$.
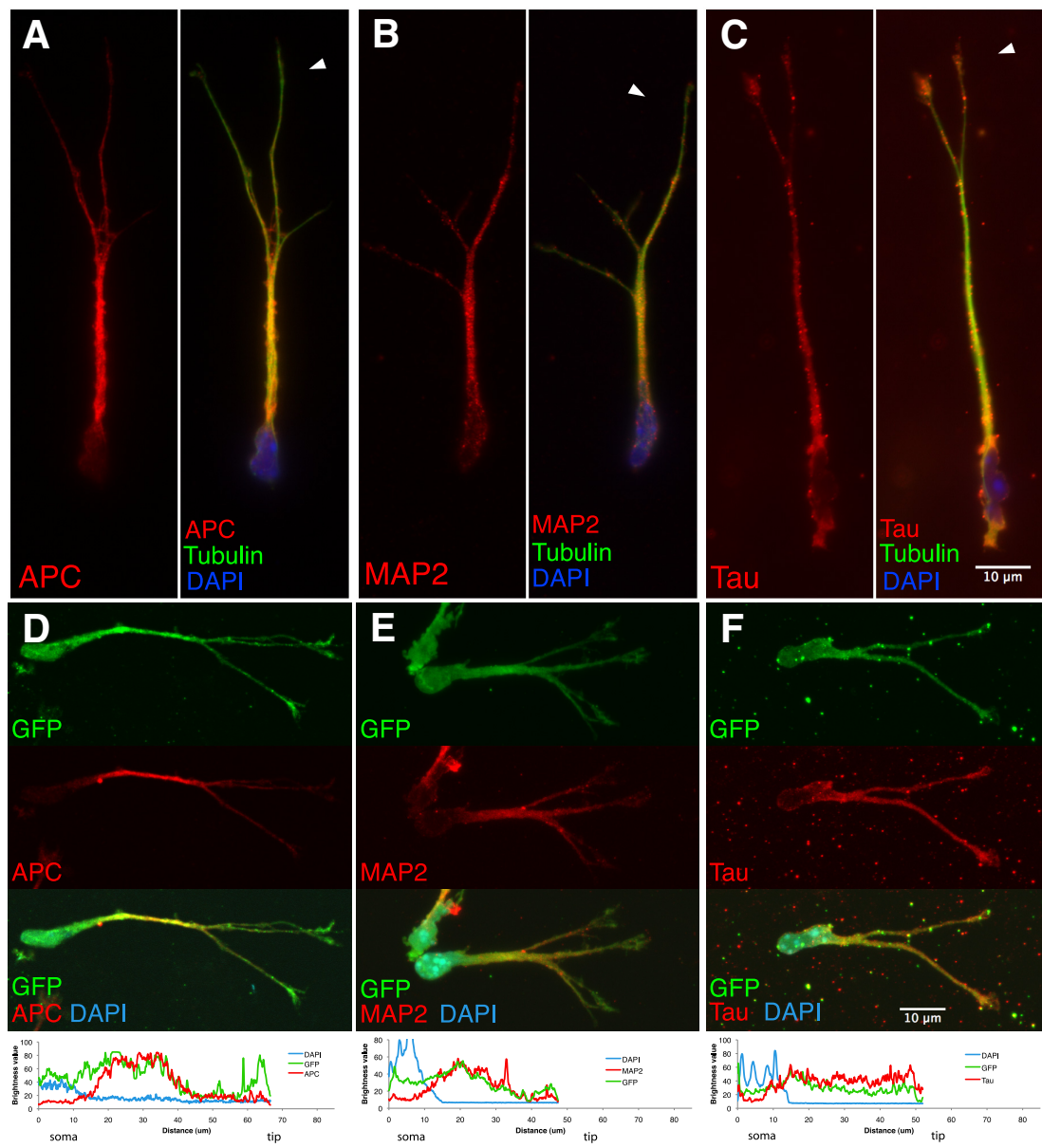

Figure 10. Different MAPs have distinct localizations in migrating interneurons. A, APC. B, MAP2.C, Tau immunolabeling in red with tubulin (green) and nuclear (DAPI, blue) labeling. APC $(\boldsymbol{A})$ and MAP2 $(\boldsymbol{B})$ decorate microtubules throughout the leading process of migrating interneurons, but are concentrated proximal to the nucleus and not at the leading process tip (arrowheads). C, Tau also decorates microtubules throughout the leading process, but is present in both the proximal and distal leading process and is present at the leading process tip (arrowhead). $\boldsymbol{D}-\boldsymbol{F}$, Immunolabeling of MAPS (red), cytosolic GFP (green), the nucleus (DAPI, blue) and quantification of MAP distribution. D, APC is not enriched in the distal leading process in $96 \%$ of interneurons. $\boldsymbol{E}$, MAP2 is not enriched in the distal leading process in $83 \%$ of interneurons. $\boldsymbol{F}$, Tau is present in the distal leading process colocalized with GFP in $70 \%$ of neurons and enriched in $30 \%$ of interneurons. Scale bar, $10 \mu \mathrm{m}$. 

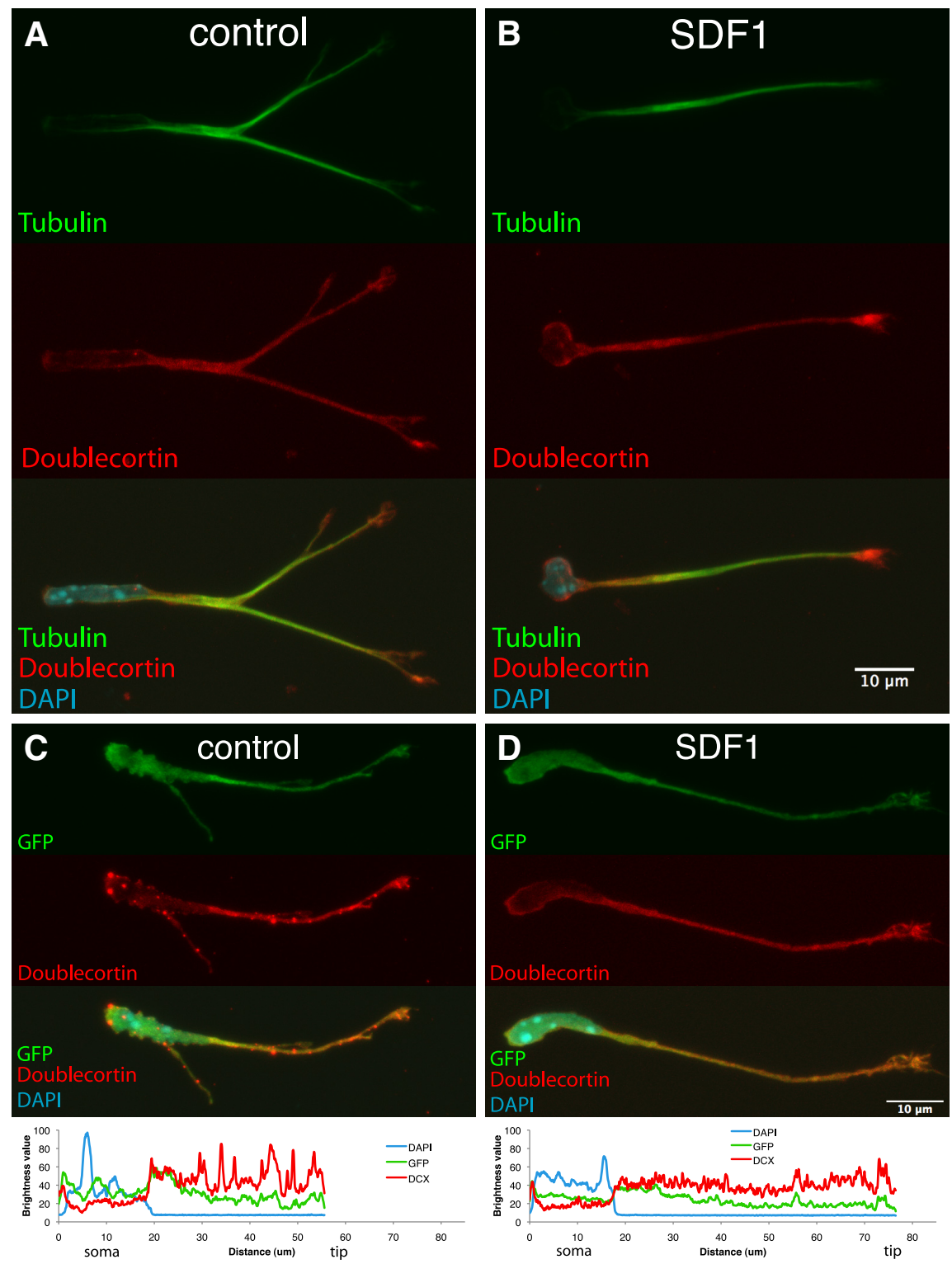

Figure 11. DCX localizes to the distal leading process in control- and SDF1-treated interneurons. $\boldsymbol{A}, \boldsymbol{B}$, Interneurons immunolabeled for tubulin (green) and DCX (red) with nuclear counterstaining (DAPI, blue). $\boldsymbol{A}, \mathrm{DCX}$ colocalizes with microtubules throughout the leading process and is enriched at the leading process tips. $\boldsymbol{B}$, SDF1 condenses leading process microtubules as seen previously while maintaining $D C X$ localization at the leading process tip. $C, D$, Control-and SDF1-treated interneurons immunolabeled for DCX and GFP (cytoplasmic marker), with quantification of the immunolabeling intensity from soma to leading process tip. In both control-and SDF1-treated interneurons, DCX is enriched in the distal leading process in $100 \%$ of interneurons. Scale bar, $10 \mu \mathrm{m}$.

1999). Plus-end binding MAPS such as EB1 are known to promote microtubule elongation, while MAPS like p150(Glued) or DCX are known to affect both microtubule nucleation and organization of the array by bundling microtubules (Gleeson et al., 1999; Ligon et al., 2003). Bundled microtubules are more stable and less likely to depolymerize and can even be resistant to the effects of the microtubule depolymerizing drug, nocodazole (Umeyama et al., 1993).

Because SDF1 reduces branching, we postulated that SDF1 would either depolymerize microtubules to collapse branches or bundle microtubules to reduce support for branches growing at angles to the main leading process. To discriminate between these two possibilities, we treated interneurons with nocodazole, a microtubule-depolymerizing drug, to test whether SDF1- regulated branches are easier to shift toward collapse. Treatment with low-dose nocodazole (10 nM) alone reduces branching frequency as expected (Fig. $9 A, B$, Movie 7). Treatment with SDF1 alone reduces branching as seen previously. If SDF1 depolymerizes microtubules, we would expect combined SDF1 and nocodazole treatment to result in extreme branching reduction. However, SDF1 treatment prevents additional nocodazole-induced collapse, indicating that SDF1 actually stabilizes the microtubule cytoskeleton (Fig. 9A,B, Movie 7). Nocodazole has a less striking effect on speed than on branching, slightly reducing migration speed. SDF1 normally increases interneuron migration speed, but low-dose nocodazole prevents this effect (Fig. 9C, Movie 7).

Given that SDF1 stabilizes the interneuron microtubule cytoskeleton but still decreases branch lifetime, we decided to examine SDF1's effect on microtubule dynamics by visualizing stable and dynamic microtubules. To visualize these two microtubule states, we immunolabeled interneurons with antibodies against acetylated (stable) and tyrosinated (dynamic) $\alpha$-tubulin (Gopal et al., 2010). In control conditions, both stable and dynamic microtubules can be seen throughout the cell; however, more of the microtubules at the leading process tip are newly polymerized, dynamic microtubules (Fig. 9D). Dynamic microtubules are also enriched at the rear of the cell and surrounding the nucleus, while the cytoplasmic bulge preceding the nucleus and the leading process shaft contain both stable and dynamic microtubules (Fig. 9D). In the presence of SDF1, the location of most dynamic and stable microtubules appears similar but the leading process becomes less branched and microtubules appear more condensed or consolidated, consistent with microtubule bundling (Fig. 9D). Short, dynamic microtubules are frequently visible protruding from the leading process shaft of SDF1-treated interneurons (Fig. 9D, arrowhead). These microtubules are thin and do not form an elaborated network at the tip of the branch as seen in normal leading process branches. Because SDF1 decreases branch lifetime, these microtubules have likely invaded the transient protrusions seen in the presence of SDF1 (Fig. 1), but do not persist long enough to become stable and support persistent branches. Treatment with 10 nM nocodazole alone reduces branching compared with control and causes microtubules to splay at the leading process tip, generating a spiky, brushy appearance (Fig. 9D). At this low dose of nocodazole dynamic microtubules continue to be enriched at the leading process tip (Fig. 9E). Combining SDF1 with $10 \mathrm{~nm}$ nocodazole does not result in collapse of the leading process and both stable and dynamic microtubules appear throughout the lead- 


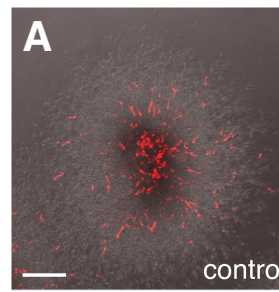

B

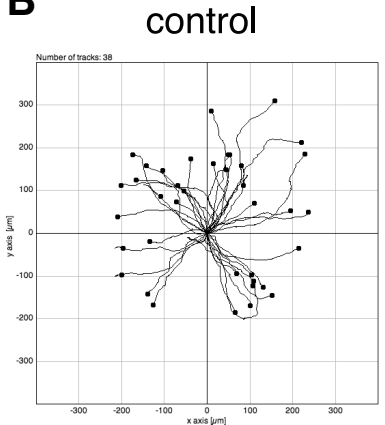

D
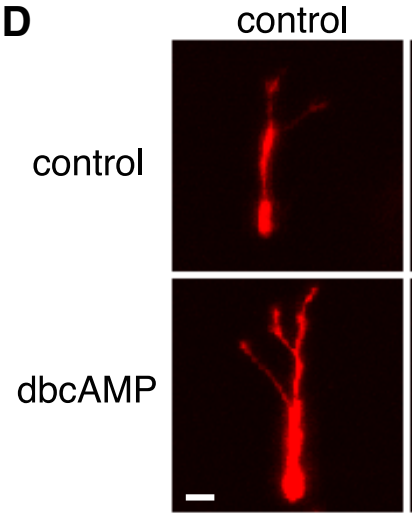

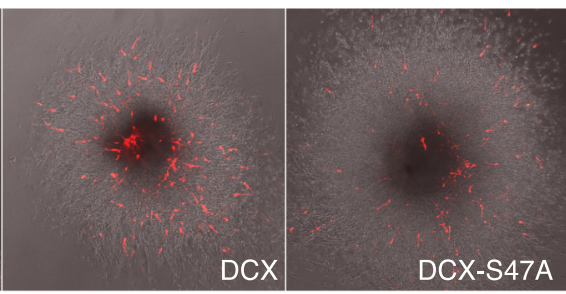

DCX
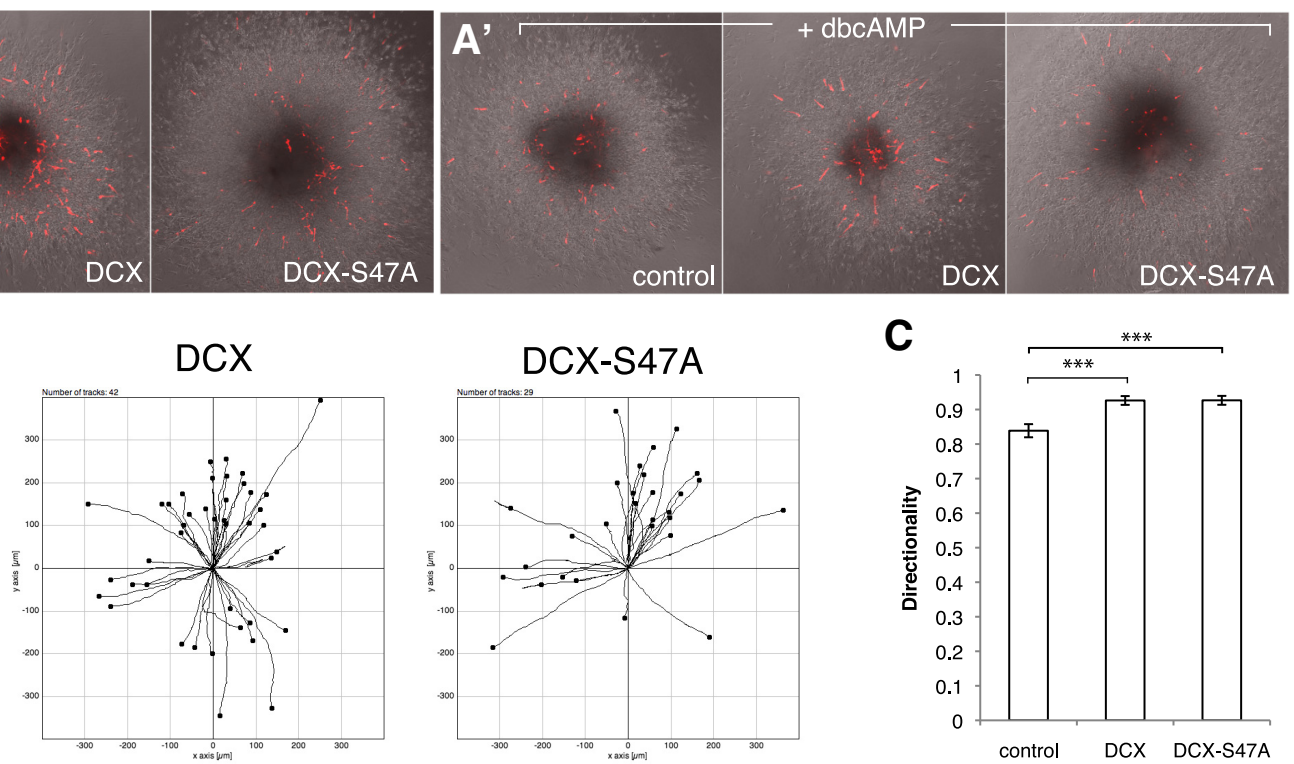

C

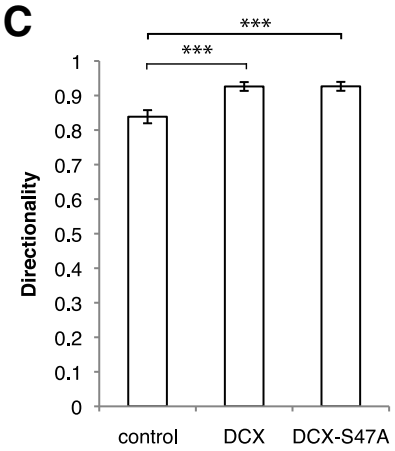

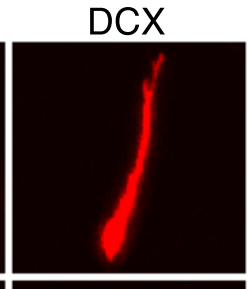

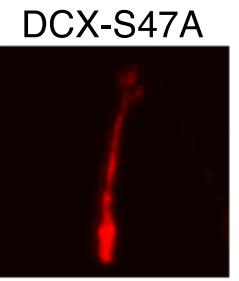

\section{E}

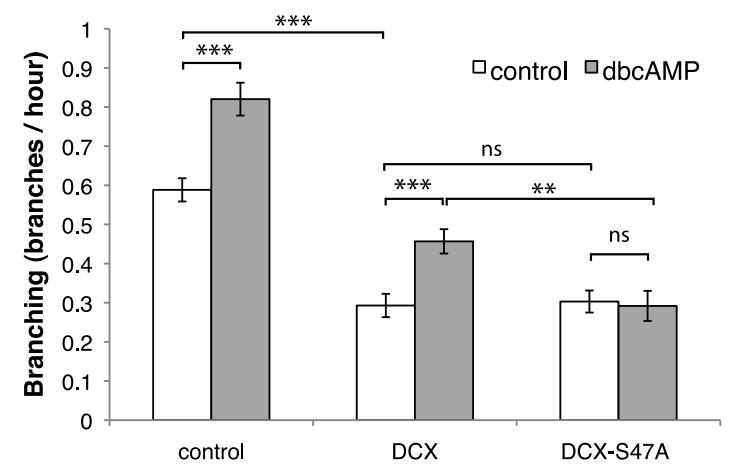

Figure 12. Interneuron branching is positively regulated by CAMP and negatively regulated by DCX. $A, \boldsymbol{A}^{\prime}$, Interneurons cotransfected with dsRed and DCX expression plasmids migrate out from medial ganglionic eminence explants (overlay of fluorescent and phase microscopy). Treatment with dbcAMP or expression of DCX or DCX-S47A does not prohibit interneuron migration. $B$, Migration path traces of processively migrating interneurons. Control interneurons typically migrate away from the explant in a curved path as they make direction changes. Interneurons overexpressing $D C X$ or DCX-S47A can migrate farther from the center of the explant and their migration paths are straighter. C, Quantification of migration path directionality, a measure of the deviation of the migration path from a straight line distance. Control interneurons migrate away from the explant while making slight changes in direction. $D C X$ or $D C X-S 47 A$ overexpressing interneurons are more likely to migrate without changing direction ( \pm SEM; ${ }^{* * *} p=0.001, t$ test). D, Frames from Movie $8 . D, E$, Compared with control, DCX or DCX-S47A overexpression reduces branching frequency. dbcAMP, a PKA agonist, increases interneuron branching. DCX overexpression's reduction in branching can be partially rescued by treatment with dbcAMP. However, dbcAMP cannot rescue branching in

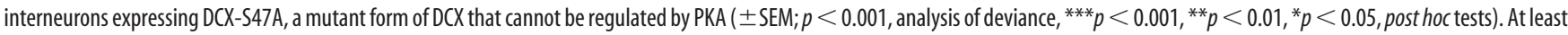
28 cells per condition, three independent experiments. Scale bars; $\boldsymbol{A}, 200 \mu \mathrm{m} ; \boldsymbol{D}, 10 \mu \mathrm{m}$.

ing process, again with dynamic microtubules enriched at the leading process tip (Fig. 9D,E). Microtubules appear condensed or consolidated, similar to SDF1 treatment alone. Together, these results indicate that SDF1 does not depolymerize microtubules, but likely activates a protein involved in microtubule stability.

\section{Microtubule-associated proteins have polarized distributions} within the interneuron leading process

In response to SDF1, we have observed distinct changes in microtubule organization in two different locations in the cell: short dynamic microtubules protruding from the leading process shaft and condensed or bundled microtubules within the leading process. Subcellular regulation of microtubules is accomplished by MAPS that bind to microtubules in different cellular domains and control microtubule dynamics through promotion of polymerization, stabilization, or bundling (Dehmelt and Halpain, 2004). Given their highly polarized morphology, MAP regulation of microtubules is especially important in neurons and several MAPS such as Tau, MAP2, APC, and DCX play distinct roles regulating microtubules in neurons. For example, Tau promotes microtubule stability and bundling in the axon of mature neurons (Caceres and Kosik, 1990; Baas et al., 1991), while MAP2 stabilizes microtubules in dendrites (Caceres et al., 1992; Chen et al., 1992). APC is thought to promote microtubule elongation in migrating excitatory neurons (Asada and Sanada, 2010), while DCX stabilizes and bundles microtubules and is localized to the tips of neurites and leading processes of migrating SVZa neurons (Gleeson et al., 1999; Schaar et al., 2004).

We hypothesize that SDF1 primarily regulates microtubule bundling near the leading process tip. To establish the subcellular localization of these MAPS in migrating interneurons, we performed coimmunolabeling of interneurons for each MAP and tubulin. All the MAPS decorated microtubules throughout the leading process; however, APC and MAP2 are highly localized proximal to the nucleus and less prominent at the leading process 
tip (Fig. 10A,B). Tau is localized throughout the leading process and is present at the leading process tip (Fig. 10C). To quantify the distribution of each MAP we compared MAP distribution to the cytosolic marker GFP. APC was the least prominent at the leading process tip in most interneurons (less than GFP in $96 \%$ of interneurons, $4 \%$ equal, $n=30$ ), while MAP2 was also not prominent at the leading process tip (less than GFP in $83 \%, 17 \%$ equal, $n=45)$. APC and MAP2 were also not prominent in the nucleus (100\% of interneurons). In the cytoplasmic bulge proximal to the nucleus APC is more prominent than GFP in $47 \%$ of interneurons and equally prominent in $53 \%$ of interneurons, while MAP2 is more prominent than GFP in $40 \%$ of neurons and equally prominent in $54 \%$ of neurons ( $6 \%$ less prominent). In contrast, Tau was equally prominent as GFP at the leading process tip in many interneurons (70\%) and more prominent in 30\%. At the cytoplasmic bulge, Tau is more prominent than GFP in $43 \%$ of neurons and equally prominent in $57 \%$ of neurons $(n=40)$. Tau is also not prominent in the nucleus in $72 \%$ of interneurons, but equal in $28 \%$.

APC's localization in these inhibitory interneurons contrasts with the previously reported localization in migrating excitatory neurons where APC decorates microtubule plus end tips at the leading process tip (Asada and Sanada, 2010). MAP2 and Tau's overlapping localization in much of the interneuron leading process contrasts with their nonoverlapping distribution in mature neurons, in which MAP2 and Tau exclusively occupy dendrites and axons, respectively. Together these results indicate that localization of some MAPS may be different in migrating interneurons than migrating excitatory neurons or stationary mature neurons.

Given these results, APC and MAP2 are not the best candidates to control branching in the distal leading process, while Tau is present in the distal leading process, although not enriched at the tip $100 \%$ of the time.

\section{DCX is localized to the distal leading process in control- and SDF1-treated interneurons}

We also investigated the subcellular localization of DCX by again performing coimmunolabeling of DCX and microtubules or cytoplasmic GFP. DCX is an excellent candidate for bundling microtubules downstream of SDF1 because DCX is regulated by PKA and knockdown of DCX results in increased branching in migrating neurons (Schaar et al., 2004; Kappeler et al., 2006). DCX localizes to the leading process and decorates microtubules at the leading process tip in both control- and SDF1- treated interneurons (Fig. 11A,B). Comparing the distribution of DCX to a cytoplasmic reference GFP, DCX is always prominent at the leading process tip (100\% of interneurons), less prominent in the nucleus ( $97 \%$ of interneurons), and equal ( $24 \%$ of interneurons) or less prominent at the cytoplasmic bulge proximal to the nucleus (76\% of interneurons, $n=42$; Fig. $11 C$ ). SDF1 treatment did not appear to change DCX localization at the leading process tip (100\% of interneurons), nucleus (less prominent, $97 \%$ of interneurons), or at the bulge (equal, $29 \%$ of interneurons; less prominent, $71 \%, n=42$; Fig. $11 D$ ). These data show that DCX is the best candidate examined to bundle and stabilize microtubules in the distal leading process and regulate interneuron branching.

DCX overexpression reduces branching and is regulated by cAMP in interneurons

DCX depletion through RNAi or genetic knock out of DCX results in interneurons that branch more frequently, making DCX an excellent candidate to mediate SDF1 branching reduction

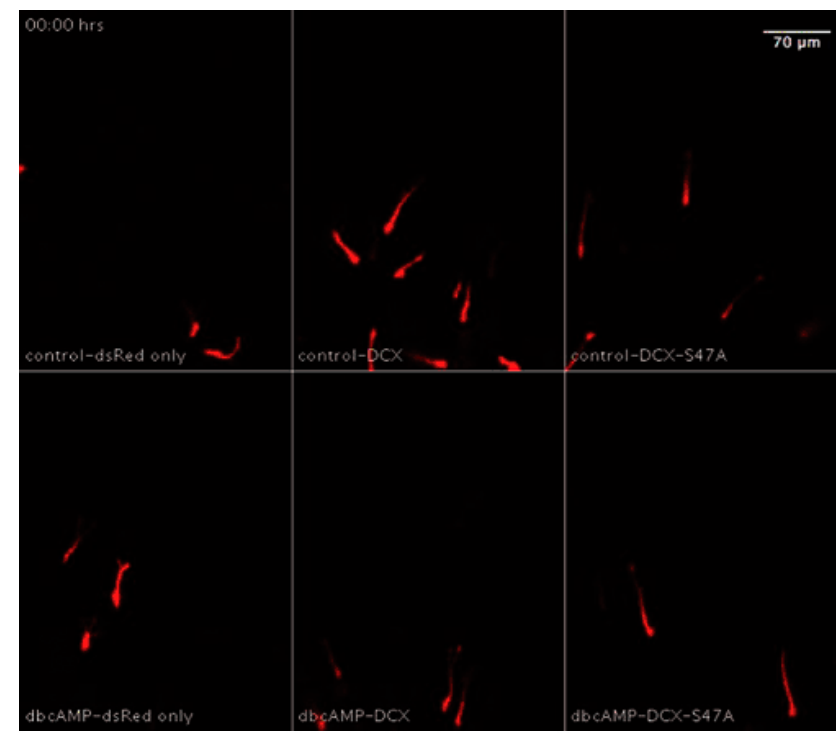

Movie 8. Interneuron branching is positively regulated by cAMP and negatively regulated by DCX. Control, Control-treated interneurons expressing dsRed only display frequent branching and normal migration. Control-DCX, Interneurons expressing DCX have a straighter morphology, branch less frequently, and have straighter migration paths. Control-DCX-S47A, Interneurons expressing DCX-S47A, a mutant form of DCX that cannot be phosphorylated by PKA, have a similar phenotype to DCX-expressing interneurons. dbcAMP-dsRed only, Interneurons treated with dbcAMP branch more frequently than control cells. dbcAMP-DCX, Treating DCX-expressing interneurons with dbcAMP increases branching frequency compared with untreated $D C X$ expressing interneurons. dbCAMP-DCX-S47A, Treating DCX-S47A-expressing interneurons with $\mathrm{dbcAMP}$ does not increase branching frequency compared with untreated DCX-expressing interneurons, indicating that CAMP regulates DCX reduction of branching through $\$ 47.20 \times$ time-lapse confocal microscopy, 1 frame/10 min.]Scale bar, $70 \mu \mathrm{m}$.

(Kappeler et al., 2006; Friocourt et al., 2007). Through its two microtubule binding domains, DCX bundles microtubules in vitro and in cultured cells (Schaar et al., 2004). To test whether DCX bundling of microtubules is sufficient to reduce branching in interneurons, we coelectroporated DCX and dsRed expression plasmids into E14.5 mouse brain slices and cultured MGE explants (Fig. 12A). We performed time-lapse imaging of interneurons as they migrated out from the center of the explant. Interneurons overexpressing DCX make straighter, more direct migration paths and their branching is reduced (Fig. 12B-D, Movie 8). Some DCX-expressing interneurons are also able to migrate much farther out of the explant center than control cells. These results indicate that DCX overexpression is sufficient to straighten the migration path of interneurons and reduce branching.

DCX is negatively regulated by phosphorylation at several regulatory serines. Serine 47 has been identified as a PKA regulatory site (Schaar et al., 2004), so we created a DCX-S47A mutant that cannot be regulated by PKA, and will mimic a nonphosphorylated DCX with high affinity to microtubules. Overexpression of DCX-S47A increases direct migration paths similar to DCX (Fig. $12 B, C)$. Importantly, overexpression of either form of DCX straightens the leading process and reduces branching (Fig. $12 \mathrm{D}, \mathrm{E}$, Movie 8), suggesting that microtubule bundling by DCX reduces branching. To test whether cAMP levels regulate DCX reduction of interneuron branching, we treated interneurons with PKA agonist dbcAMP. Treatment with dbcAMP increases branching in control interneurons, and can partially rescue the effect of expressing DCX (Fig. 12D,E), indicating that cAMP represses DCX branching reduction in interneurons. Treat- 
ment with dbcAMP cannot rescue branching reduction in interneurons expressing DCX-S47A (Fig. 12D,E), indicating that CAMP-dependent repression of DCX activity occurs through serine 47 in DCX, a known PKA regulatory site. These results indicate that cAMP is an important regulator of DCX's ability to bundle microtubules and decrease interneuron branching.

\section{DCX is required for SDF1 reduction of branching}

We have previously shown that SDF1 regulates interneuron migration through the G-protein $\mathrm{G}_{\mathrm{i}}$ (Lysko et al., 2011). $\mathrm{G}_{\mathrm{i}}$ typically reduces cAMP levels and PKA activity, suggesting that SDF1 would result in DCX activation and bundling of microtubules thereby reducing interneuron branching. We hypothesized that SDF1 increases the amount of DCX with high microtubule bundling activity to ultimately reduce interneuron branching. To test whether SDF1 requires DCX to inhibit branching we performed knockdown experiments of DCX in the presence and absence of SDF1. We used short hairpin RNAi (shRNA) constructs targeted to the $3^{\prime}$ untranslated region of endogenous DCX mRNA, allowing rescue with plasmids expressing only the coding region of DCX (Bai et al., 2003; Ramos et al., 2006).

DCX knockdown results in an increase in interneuron branching, as seen previously (Fig. 13 A, B, Movie 9; Friocourt et al., 2007). SDF1 treatment normally decreases branching in control interneurons; however, when DCX is knocked down, SDF1 cannot fully reduce interneuron branching (Fig. $13 A, B$ ). This indicates that DCX is required for SDF1 reduction of branching.

The increase in branching seen upon DCX knockdown can be rescued by DCX or DCX-S47A expression. Rescuing with normal DCX or DCX-S47A results in a reduced level of branching compared with control interneurons, but largely normal migration (Fig. 13 A, B, Movie 9). Under normal DCX rescue conditions, SDF1 can reduce branching. Under DCX-S47A rescue conditions, SDF1 further reduces branching. These data are consistent with the idea that when SDF1 signaling cannot further activate DCX-S47A (already active bundling microtubules and reducing branching), SDF1 signaling will reduce branching further through the alternative pathway of cortactin downregulation. Together, these data indicate that DCX is required for full SDF1driven reduction in interneuron branching, and suggest that SDF1 signaling activates DCX in combination with downregulation of cortactin to reduce interneuron branching.

\section{Discussion}

In this study we sought to understand the mechanism by which SDF1 regulates leading process branching. We identified two cytoskeletal regulators downstream of SDF1: cortactin, which regulates actin, and DCX, which regulates microtubules. Based on these data we developed a model to explain how regulating both actin and microtubule networks may affect interneuron branching (Fig. 14). Under basal branching conditions F-actin is enriched at the tip of the leading process and is arranged in a highly branched network created by cortactin, which along with Arp2/3 is known to promote branch growth in neurons (Mingorance-Le Meur and O'Connor, 2009; Spillane et al., 2011; Hu et al., 2012). Microtubule invasion changes growing actin protrusions to stable branches.

Upon SDF1 signaling, calpain protease becomes active and cleaves cortactin, reducing Arp2/3 activity, consolidating the actin network and reducing branch lifetime. SDF1 signaling simultaneously activates DCX to bind and bundle microtubules, stabilizing the microtubule array and reducing support of offangle branches. Although not directly addressed in our study, in addition to bundling microtubules, DCX is a component of nuclear movement and also promotes microtubule elongation (Taylor et al., 2000; Tanaka et al., 2004b). The combination of actin network consolidation and DCX activation suggests a mechanism for how SDF1 simultaneously reduces branching while increasing migration speed.

Because DCX is localized to the distal leading process, bundling of microtubules is most efficient at the leading process tip, promoting forward migration. More proximal to the nucleus, some microtubules may become unbundled and invade newly formed protrusions, giving rise to the short-lived branches we see 


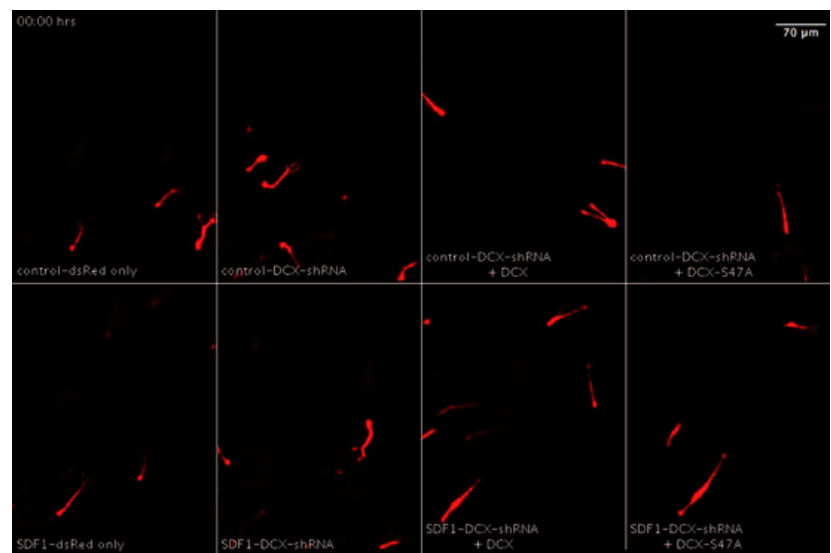

Movie 9. Knockdown of DCX causes increased interneuron branching and blocks SDF1reduction of branching. Control-dsRed only, Control-treated interneurons display frequent branching. Control-DCX-shRNA, Interneurons expressing DCX-shRNA branch more frequently than control interneurons, consistent with $D C X$ normally limiting branching. Control-DCXshRNA + DCX, The increase in branching by DCX knockdown (targeted to $3^{\prime}$-UTR) can be rescued by expressing DCX (coding sequence only). Interneurons display more normal migration and branch slightly less than control interneurons. Control-DCX-shRNA + DCX-S47A, Interneurons rescued with the $S 47 A$ mutant form of DCX display similar migration and branching phenotype as interneurons rescued with normal DCX. SDF1-dsRed only, SDF1 reduces interneuron branching frequency. SDF1-DCX-shRNA, SDF1 does not reduce branching in DCX-shRNA-expressing interneurons. SDF1-DCX-shRNA + DCX, SDF1 reduces branching in DCX knockdown + rescue interneurons. SDF1-DCX-shRNA + DCX-S47A, SDF1 further reduces branching in interneurons rescued with the S47A mutant form of DCX. $20 X$ time-lapse confocal microscopy, 1 frame/10 min. Scale bar, $70 \mu \mathrm{m}$.

in the presence of SDF1 (Figs. 1, 8). However, reduced cortactinArp $2 / 3$ activity and less stabilized microtubules lead to quick collapse of these branches.

This model is consistent with previous studies indicating that DCX is necessary for limiting branching in neurons (Kappeler et al., 2006; Bielas et al., 2007; Friocourt et al., 2007). In this paper we provide evidence that overexpression of DCX is sufficient to reduce branching and affect the migration path of interneurons. We also show that cAMP increases interneuron branching frequency and negatively regulates DCX reduction of branching through serine 47. DCX's serine 47 was previously identified as a PKA/MARK-regulated serine that, when phosphorylated, reduces DCX microtubule bundling (Schaar et al., 2004). These authors suggested that unknown signaling could act through serine 47 to influence neuronal migration. In this study we identify DCX as a downstream effector of SDF1, linking an extracellular guidance factor to this cytoskeletal regulatory protein.

Previous studies have established that DCX localization to the tips of axons and neurites is dependent on protein phosphatase activity (Schaar et al., 2004; Bielas et al., 2007). Inhibiting this phosphatase activity changes the localization of DCX from tips of neurites to the cell soma. In our study, we do find that DCX is localized to the distal leading process, but do not find evidence that SDF1 signaling changes DCX localization. This may indicate that SDF1 represses kinases whose activity is uniform throughout the leading process, while polarized phosphatases maintain DCX localization. Minimal changes in DCX localization are expected as both control- and SDF1-treated interneurons maintain limited branching and forward migration, consistent with the idea that SDF1 only fine-tunes interneuron branching.

SDF1 signaling affects the migration of diverse cell types like human leukocytes and zebrafish germ cells, and controls the process of neuronal axon pathfinding (Bleul et al., 1996; Chalasani et al., 2003; Knaut et al., 2003). In leukocytes, germ cells, and migrating interneurons, the data support a $\mathrm{G}_{\mathrm{i}} \alpha$ signal downstream of CXCR4, reducing cAMP to affect migration (Sotsios et al., 1999; Dumstrei et al., 2004; Dong et al., 2006; Lysko et al., 2011). However, in studies of SDF1's role as an anti-repellent during axon guidance, at the intersection of SDF1 and slit or semaphorin signaling, while SDF1 signaling requires $G_{i}, c A M P$ levels actually increase upon SDF1 treatment as a result of Gq and $\beta \gamma$ signaling (Chalasani et al., 2003; Xu et al., 2010). This difference in SDF1 signaling pathways is accompanied by a morphological difference: SDF1 alone has no obvious effect on the morphology of sensory axon growth cones or their cytoskeleton (Twery and Raper, 2011), while we demonstrate that SDF1 changes the organization of both actin and microtubules while reducing branching. These differences suggest that SDF1's signaling pathway may vary between migrating interneurons and sensory axon pathfinding, underscoring the cell type-specific nature of signal transduction.

Our data are consistent with the signaling pathway regulating branching of developing hippocampal neurons in culture. NT3 or BDNF increase cAMP in hippocampal neurons, increasing branching through calpain regulation of cortactin (Mingorance-Le Meur and O'Connor, 2009). Together our results suggest that neuronal branching can be increased or decreased by different guidance ligands using opposing G- $\alpha$ protein activities to tune cAMP levels. Recent evidence indicates that DCX activity can also be downregulated by Gs/cAMP at serine 47 in neural progenitor cells (Toriyama et al., 2012). These data complement our results indicating that CAMP regulation of serine 47 regulates interneuron branching.

We can integrate our findings in this study with previous work about the guidance of interneurons along their migration path from ventral forebrain to developing neocortex. SDF1 expressed in the SVZ/IZ regulates stream migration and the transition to cortical plate invasion (López-Bendito et al., 2008; Lysko et al., 2011). As interneurons migrate into the SDF1-rich SVZ/IZ, we expect calpain activity will increase, proteolyzing cortactin and consolidating the actin network. Less available nucleation from cortactin/Arp2/3 increases the chance that new branches will collapse and the cell will continue migrating within the SVZ/IZ stream. DCX bundling activity will prevent microtubule support for off-angle branches. As interneurons migrate to zones with lower levels of SDF1, calpain and DCX activity will decrease and cortactin will promote increased branching. This increase in branching will be supported by microtubules no longer tightly bundled by DCX, and allow interneurons to turn and invade the cortical plate. Having this more complete understanding of interneuron branching will inform future studies directed at therapy. For example, a phenotype of the CXCR4 knock-out mouse is premature cortical plate invasion, resulting in a global mislocalization of interneurons leading to altered inhibitory tone (Li et al., 2008). If suppressing branching prevented premature cortical plate invasion and rescued normal interneuron localization, normal inhibition could potentially be restored.

Mutations in DCX also cause lissencephaly associated with intellectual disabilities and epilepsy (Kerjan and Gleeson, 2007; Leger et al., 2008). Many mutations cluster in DCX's tubulin binding domains (Sapir et al., 2000; Taylor et al., 2000). JNK and Cdk5 kinases regulate several serines on DCX (Gdalyahu et al., 2004; Tanaka et al., 2004a), while serine 47 has been identified as both a MARK and PKA phosphorylation site, as well as being a mutated amino acid in patients with lissencephaly (Sapir et al., 2000; Schaar et al., 2004). If interneurons in patients carrying an 
A

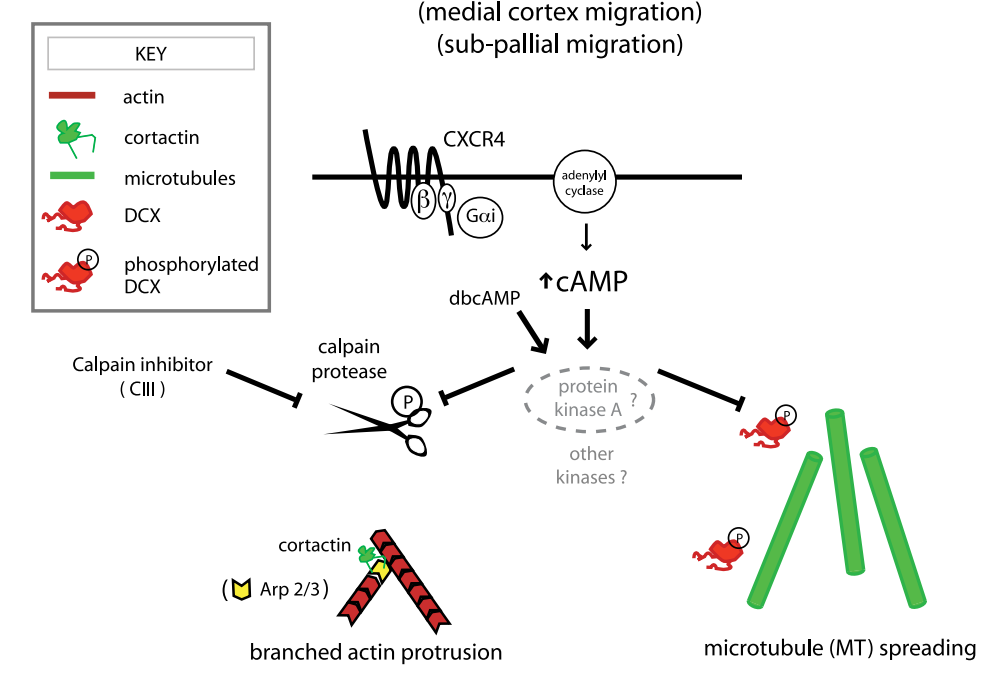

B $\quad+$ SDF1

(lateral cortex migration) (stream migration [SVZ/IZ])

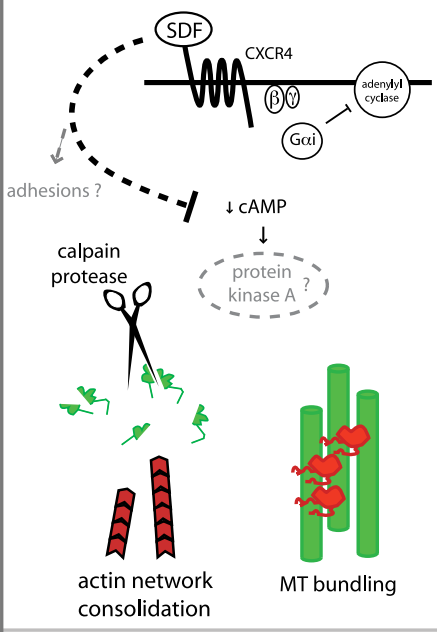

B' less branching

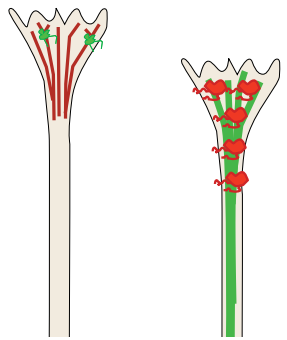

Figure 14. SDF1 coregulates both actin and microtubules to reduce interneuron branching. $A$, Model for cytoskeletal regulation under control conditions. Under control conditions adenylyl cyclase produces basal levels of cAMP, stimulating repression of both calpain protease and DCX (putatively through PKA). Cortactin is free to help Arp2/3 promote membrane protrusion and branch growth. Phosphorylation lowers DCX's affinity for microtubules, allowing the microtubule network to spread and support new branches. $A^{\prime}$, Diagram showing how high amounts of cortactin promote a branched actin network supporting interneuron branch growth. Microtubules are free to invade off-angle branches allowing migration direction to change. $\boldsymbol{B}$, Model for SDF1 regulation of the interneuron cytoskeleton. SDF1 binds to $C X C R 4$, allowing $\mathrm{G}_{\mathrm{i}}$ to inhibit adenylyl cyclase. CAMP levels drop, de-repressing calpain protease and DCX. Calpain proteolyzes cortactin and the actin network consolidates, leading to shorter lived branches. DCX affinity for microtubules increases, allowing DCX to bundle microtubules. Bundled microtubules are less likely to invade and support new branches. $\boldsymbol{B}^{\prime}$, Diagram showing how low levels of cortactin could result in a consolidated actin network. Active DCX bundles microtubules, promoting a straighter leading process, less branching support, and a direct migration path.

S47 mutation are less sensitive to SDF1, we would expect deficits in interneuron migration similar to those seen in the CXCR4-/mice (Li et al., 2008). Further investigation into how SDF1 and other guidance factors regulate DCX will be critical to understanding the consequences of different DCX mutations in patients.

In addition to guidance factors regulating cytoskeletal organization, cell adhesion is another important component in neuronal migration (Fishell and Hatten, 1991; Denaxa et al., 2001; Gupta et al., 2003; Elias et al., 2007; Shieh et al., 2011). While we have focused on calpain cleavage of cortactin, calpains are also known to cleave components of cell adhesions such as talin or paxillin and regulatory proteins such as focal adhesion kinase (Franco and Huttenlocher, 2005). When we inhibited calpain in migrating neurons, both branching and migration speed were reduced, despite the increase in actin at the leading process tip (Fig. 5). Similarly, embryonic fibroblasts deficient in Calpain4 do not spread or migrate well, despite increased filopodial protrusiveness (Dourdin et al., 2001; Perrin et al., 2006). Inhibiting calpain protease can increase (Lokuta et al., 2003) or decrease cell migration (Dourdin et al., 2001) depending on conditions and cell type, supporting the idea that calpain protease can impact the amount of adhesion turnover optimal for migration. We have hypothesized that in calpain-inhibited interneurons, nonoptimal adhesion turnover decreases branching and speed, while reduced proteolysis of cortactin increases actin at the leading process tip causing extreme protrusion (Fig. 5). Determining whether SDF1 regulates components of adhesions such as paxillin or talin is important future studies and could provide a common point of interneuron branching regulation.

Our data have advanced the understanding of the regulation of cytoskeletal dynamics in the leading process of migrating interneurons. We have established cortactin as a novel regulator of interneuron branching and identified cortactin and DCX as two downstream effectors of SDF1 signaling. We have also described how calpain protease and CAMP are important regulators of interneuron branching. Our model describes how a guidance factor can ultimately regulate both actin and microtubules and illustrates how interneurons may use extracellular cues to change their migratory behavior, successfully migrating through the developing neocortex. 


\section{References}

Asada N, Sanada K (2010) LKB1-mediated spatial control of GSK3beta and adenomatous polyposis coli contributes to centrosomal forward movement and neuronal migration in the developing neocortex. J Neurosci 30:8852-8865. CrossRef Medline

Baas PW, Pienkowski TP, Kosik KS (1991) Processes induced by tau expression in Sf9 cells have an axon-like microtubule organization. J Cell Biol 115:1333-1344. CrossRef Medline

Bai J, Ramos RL, Ackman JB, Thomas AM, Lee RV, LoTurco JJ (2003) RNAi reveals doublecortin is required for radial migration in rat neocortex. Nat Neurosci 6:1277-1283. CrossRef Medline

Baudoin JP, Alvarez C, Gaspar P, Métin C (2008) Nocodazole-induced changes in microtubule dynamics impair the morphology and directionality of migrating medial ganglionic eminence cells. Dev Neurosci 30:132143. CrossRef Medline

Bellion A, Baudoin JP, Alvarez C, Bornens M, Métin C (2005) Nucleokinesis in tangentially migrating neurons comprises two alternating phases: forward migration of the Golgi/centrosome associated with centrosome splitting and myosin contraction at the rear. J Neurosci 25:5691-5699. CrossRef Medline

Bielas SL, Serneo FF, Chechlacz M, Deerinck TJ, Perkins GA, Allen PB, Ellisman MH, Gleeson JG (2007) Spinophilin facilitates dephosphorylation of doublecortin by PP1 to mediate microtubule bundling at the axonal wrist. Cell 129:579-591. CrossRef Medline

Bleul CC, Fuhlbrigge RC, Casasnovas JM, Aiuti A, Springer TA (1996) A highly efficacious lymphocyte chemoattractant, stromal cell-derived factor 1 (SDF-1). J Exp Med 184:1101-1109. CrossRef Medline

Burkel BM, von Dassow G, Bement WM (2007) Versatile fluorescent probes for actin filaments based on the actin-binding domain of utrophin. Cell Motil Cytoskeleton 64:822-832. CrossRef Medline

Caceres A, Kosik KS (1990) Inhibition of neurite polarity by tau antisense oligonucleotides in primary cerebellar neurons. Nature 343:461-463. CrossRef Medline

Caceres A, Mautino J, Kosik KS (1992) Suppression of MAP2 in cultured cerebellar macroneurons inhibits minor neurite formation. Neuron 9:607-618. CrossRef Medline

Cassimeris L (1999) Accessory protein regulation of microtubule dynamics throughout the cell cycle. Curr Opin Cell Biol 11:134-141. CrossRef Medline

Chalasani SH, Sabelko KA, Sunshine MJ, Littman DR, Raper JA (2003) A chemokine, SDF-1, reduces the effectiveness of multiple axonal repellents and is required for normal axon pathfinding. J Neurosci 23:1360-1371. Medline

Chen J, Kanai Y, Cowan NJ, Hirokawa N (1992) Projection domains of MAP2 and tau determine spacings between microtubules in dendrites and axons. Nature 360:674-677. CrossRef Medline

D'Agostino MD, Bernasconi A, Das S, Bastos A, Valerio RM, Palmini A, Costa da Costa J, Scheffer IE, Berkovic S, Guerrini R, Dravet C, Ono J, Gigli G, Federico A, Booth F, Bernardi B, Volpi L, Tassinari CA, Guggenheim MA, Ledbetter DH, et al. (2002) Subcortical band heterotopia (SBH) in males: clinical, imaging and genetic findings in comparison with females. Brain 125:2507-2522. CrossRef Medline

Debes GF, Dahl ME, Mahiny AJ, Bonhagen K, Campbell DJ, Siegmund K, Erb KJ, Lewis DB, Kamradt T, Hamann A (2006) Chemotactic responses of IL-4-, IL-10-, and IFN-gamma-producing CD4 + T cells depend on tissue origin and microbial stimulus. J Immunol 176:557-566. Medline

de Forges H, Bouissou A, Perez F (2012) Interplay between microtubule dynamics and intracellular organization. Int J Biochem Cell Biol 44:266274. CrossRef Medline

Dehmelt L, Halpain S (2004) Actin and microtubules in neurite initiation: are MAPs the missing link? J Neurobiol 58:18-33. CrossRef Medline

Denaxa M, Chan CH, Schachner M, Parnavelas JG, Karagogeos D (2001) The adhesion molecule TAG-1 mediates the migration of cortical interneurons from the ganglionic eminence along the corticofugal fiber system. Development 128:4635-4644. Medline

Dent EW, Kalil K (2001) Axon branching requires interactions between dynamic microtubules and actin filaments. J Neurosci 21:9757-9769. Medline

Dong H, Osmanova V, Epstein PM, Brocke S (2006) Phosphodiesterase 8 (PDE8) regulates chemotaxis of activated lymphocytes. Biochem Biophys Res Commun 345:713-719. CrossRef Medline

Dourdin N, Bhatt AK, Dutt P, Greer PA, Arthur JS, Elce JS, Huttenlocher A
(2001) Reduced cell migration and disruption of the actin cytoskeleton in calpain-deficient embryonic fibroblasts. J Biol Chem 276:4838248388. Medline

Dumstrei K, Mennecke R, Raz E (2004) Signaling pathways controlling primordial germ cell migration in zebrafish. J Cell Sci 117:4787-4795. CrossRef Medline

Elias LA, Wang DD, Kriegstein AR (2007) Gap junction adhesion is necessary for radial migration in the neocortex. Nature 448:901-907. CrossRef Medline

Fishell G, Hatten ME (1991) Astrotactin provides a receptor system for CNS neuronal migration. Development 113:755-765. Medline

Flames N, Long JE, Garratt AN, Fischer TM, Gassmann M, Birchmeier C, Lai C, Rubenstein JL, Marín O (2004) Short- and long-range attraction of cortical GABAergic interneurons by neuregulin-1. Neuron 44:251-261. CrossRef Medline

Franco SJ, Huttenlocher A (2005) Regulating cell migration: calpains make the cut. J Cell Sci 118:3829-3838. CrossRef Medline

Franco SJ, Rodgers MA, Perrin BJ, Han J, Bennin DA, Critchley DR, Huttenlocher A (2004) Calpain-mediated proteolysis of talin regulates adhesion dynamics. Nat Cell Biol 6:977-983. CrossRef Medline

Friocourt G, Liu JS, Antypa M, Rakic S, Walsh CA, Parnavelas JG (2007) Both doublecortin and doublecortin-like kinase play a role in cortical interneuron migration. J Neurosci 27:3875-3883. CrossRef Medline

Friocourt G, Marcorelles P, Saugier-Veber P, Quille ML, Marret S, Laquerrière A (2011) Role of cytoskeletal abnormalities in the neuropathology and pathophysiology of type I lissencephaly. Acta Neuropathol 121:149170. CrossRef Medline

Gdalyahu A, Ghosh I, Levy T, Sapir T, Sapoznik S, Fishler Y, Azoulai D, Reiner O (2004) DCX, a new mediator of the JNK pathway. EMBO J 23:823832. CrossRef Medline

Gibson DA, Ma L (2011) Developmental regulation of axon branching in the vertebrate nervous system. Development 138:183-195. CrossRef Medline

Gleeson JG, Lin PT, Flanagan LA, Walsh CA (1999) Doublecortin is a microtubule-associated protein and is expressed widely by migrating neurons. Neuron 23:257-271. CrossRef Medline

Gopal PP, Simonet JC, Shapiro W, Golden JA (2010) Leading process branch instability in Lis1 \pm nonradially migrating interneurons. Cereb Cortex 20:1497-1505. CrossRef Medline

Gupta A, Sanada K, Miyamoto DT, Rovelstad S, Nadarajah B, Pearlman AL, Brunstrom J, Tsai LH (2003) Layering defect in p35 deficiency is linked to improper neuronal-glial interaction in radial migration. Nat Neurosci 6:1284-1291. CrossRef Medline

Hu J, Bai X, Bowen JR, Dolat L, Korobova F, Yu W, Baas PW, Svitkina T, Gallo G, Spiliotis ET (2012) Septin-driven coordination of actin and microtubule remodeling regulates the collateral branching of axons. Curr Biol 22:1109-1115. CrossRef Medline

Huang Y, Comiskey EO, Dupree RS, Li S, Koleske AJ, Burkhardt JK (2008) The c-Abl tyrosine kinase regulates actin remodeling at the immune synapse. Blood 112:111-119. CrossRef Medline

Kalil K, Szebenyi G, Dent EW (2000) Common mechanisms underlying growth cone guidance and axon branching. J Neurobiol 44:145-158. CrossRef Medline

Kappeler C, Saillour Y, Baudoin JP, Tuy FP, Alvarez C, Houbron C, Gaspar P, Hamard G, Chelly J, Métin C, Francis F (2006) Branching and nucleokinesis defects in migrating interneurons derived from doublecortin knockout mice. Hum Mol Genet 15:1387-1400. CrossRef Medline

Kerjan G, Gleeson JG (2007) Genetic mechanisms underlying abnormal neuronal migration in classical lissencephaly. Trends Genet 23:623-630. CrossRef Medline

Knaut H, Werz C, Geisler R, Nüsslein-Volhard C (2003) A zebrafish homologue of the chemokine receptor Cxcr4 is a germ-cell guidance receptor. Nature 421:279-282. CrossRef Medline

Leger PL, Souville I, Boddaert N, Elie C, Pinard JM, Plouin P, Moutard ML, des Portes V, Van Esch H, Joriot S, Renard JL, Chelly J, Francis F, Beldjord C, Bahi-Buisson N (2008) The location of DCX mutations predicts malformation severity in X-linked lissencephaly. Neurogenetics 9:277-285. CrossRef Medline

Li G, Adesnik H, Li J, Long J, Nicoll RA, Rubenstein JL, Pleasure SJ (2008) Regional distribution of cortical interneurons and development of inhibitory tone are regulated by Cxcl12/Cxcr4 signaling. J Neurosci 28:10851098. CrossRef Medline 
Ligon LA, Shelly SS, Tokito M, Holzbaur EL (2003) The microtubule plusend proteins EB1 and dynactin have differential effects on microtubule polymerization. Mol Biol Cell 14:1405-1417. CrossRef Medline

Lin F, Butcher EC (2006) T cell chemotaxis in a simple microfluidic device. Lab Chip 6:1462-1469. CrossRef Medline

Lokuta MA, Nuzzi PA, Huttenlocher A (2003) Calpain regulates neutrophil chemotaxis. Proc Natl Acad Sci U S A 100:4006-4011. CrossRef Medline

López-Bendito G, Sánchez-Alcañiz JA, Pla R, Borrell V, Picó E, Valdeolmillos M, Marín O (2008) Chemokine signaling controls intracortical migration and final distribution of GABAergic interneurons. J Neurosci 28: 1613-1624. CrossRef Medline

Lysko DE, Putt M, Golden JA (2011) SDF1 regulates leading process branching and speed of migrating interneurons. J Neurosci 31:17391745. CrossRef Medline

Marín O, Rubenstein JL (2003) Cell migration in the forebrain. Annu Rev Neurosci 26:441-483. CrossRef Medline

Martini FJ, Valiente M, López Bendito G, Szabó G, Moya F, Valdeolmillos M, Marín O (2009) Biased selection of leading process branches mediates chemotaxis during tangential neuronal migration. Development 136:4150. CrossRef Medline

Matsumoto N, Leventer RJ, Kuc JA, Mewborn SK, Dudlicek LL, Ramocki MB, Pilz DT, Mills PL, Das S, Ross ME, Ledbetter DH, Dobyns WB (2001) Mutation analysis of the DCX gene and genotype/phenotype correlation in subcortical band heterotopia. Eur J Hum Genet 9:5-12. CrossRef Medline

McManus MF, Nasrallah IM, Pancoast MM, Wynshaw-Boris A, Golden JA (2004) Lis1 is necessary for normal non-radial migration of inhibitory interneurons. Am J Pathol 165:775-784. CrossRef Medline

Métin C, Baudoin JP, Rakić S, Parnavelas JG (2006) Cell and molecular mechanisms involved in the migration of cortical interneurons. Eur J Neurosci 23:894-900. CrossRef Medline

Mingorance-Le Meur A, O'Connor TP (2009) Neurite consolidation is an active process requiring constant repression of protrusive activity. EMBO J 28:248-260. CrossRef Medline

Perrin BJ, Amann KJ, Huttenlocher A (2006) Proteolysis of cortactin by calpain regulates membrane protrusion during cell migration. Mol Biol Cell 17:239-250. Medline

Pilz DT, Matsumoto N, Minnerath S, Mills P, Gleeson JG, Allen KM, Walsh CA, Barkovich AJ, Dobyns WB, Ledbetter DH, Ross ME (1998) LIS1 and XLIS (DCX) mutations cause most classical lissencephaly, but different patterns of malformation. Hum Mol Genet 7:2029-2037. CrossRef Medline

Ramos RL, Bai J, LoTurco JJ (2006) Heterotopia formation in rat but not mouse neocortex after RNA interference knockdown of DCX. Cereb Cortex 16:1323-1331. Medline

Ridley AJ (2011) Life at the leading edge. Cell 145:1012-1022. CrossRef Medline

Sánchez-Alcañiz JA, Haege S, Mueller W, Pla R, Mackay F, Schulz S, LópezBendito G, Stumm R, Marín O (2011) Cxcr7 controls neuronal migration by regulating chemokine responsiveness. Neuron 69:77-90. CrossRef Medline

Sapir T, Horesh D, Caspi M, Atlas R, Burgess HA, Wolf SG, Francis F, Chelly J, Elbaum M, Pietrokovski S, Reiner O (2000) Doublecortin mutations cluster in evolutionarily conserved functional domains. Hum Mol Genet 9:703-712. CrossRef Medline

Schaar BT, McConnell SK (2005) Cytoskeletal coordination during neuronal migration. Proc Natl Acad Sci U S A 102:13652-13657. CrossRef Medline

Schaar BT, Kinoshita K, McConnell SK (2004) Doublecortin microtubule affinity is regulated by a balance of kinase and phosphatase activity at the leading edge of migrating neurons. Neuron 41:203-213. CrossRef Medline

Shieh JC, Schaar BT, Srinivasan K, Brodsky FM, McConnell SK (2011) En- docytosis regulates cell soma translocation and the distribution of adhesion proteins in migrating neurons. PLoS One 6:e17802. CrossRef Medline

Solecki DJ, Trivedi N, Govek EE, Kerekes RA, Gleason SS, Hatten ME (2009) Myosin II motors and F-actin dynamics drive the coordinated movement of the centrosome and soma during CNS glial-guided neuronal migration. Neuron 63:63-80. CrossRef Medline

Sotsios Y, Whittaker GC, Westwick J, Ward SG (1999) The CXC chemokine stromal cell-derived factor activates a Gi-coupled phosphoinositide 3-kinase in T lymphocytes. J Immunol 163:5954-5963. Medline

Spillane M, Ketschek A, Jones SL, Korobova F, Marsick B, Lanier L, Svitkina T, Gallo G (2011) The actin nucleating Arp $2 / 3$ complex contributes to the formation of axonal filopodia and branches through the regulation of actin patch precursors to filopodia. Dev Neurobiol 71:747-758. CrossRef Medline

Stenman J, Toresson H, Campbell K (2003) Identification of two distinct progenitor populations in the lateral ganglionic eminence: implications for striatal and olfactory bulb neurogenesis. J Neurosci 23:167-174. Medline

Stumm RK, Zhou C, Ara T, Lazarini F, Dubois-Dalcq M, Nagasawa T, Höllt V, Schulz S (2003) CXCR4 regulates interneuron migration in the developing neocortex. J Neurosci 23:5123-5130. Medline

Stumm R, Kolodziej A, Schulz S, Kohtz JD, Höllt V (2007) Patterns of SDFlalpha and SDF-1gamma mRNAs, migration pathways, and phenotypes of CXCR4-expressing neurons in the developing rat telencephalon. J Comp Neurol 502:382-399. CrossRef Medline

Tanaka T, Serneo FF, Tseng HC, Kulkarni AB, Tsai LH, Gleeson JG (2004a) Cdk5 phosphorylation of doublecortin ser297 regulates its effect on neuronal migration. Neuron 41:215-227. CrossRef Medline

Tanaka T, Serneo FF, Higgins C, Gambello MJ, Wynshaw-Boris A, Gleeson JG (2004b) Lis1 and doublecortin function with dynein to mediate coupling of the nucleus to the centrosome in neuronal migration. J Cell Biol 165:709-721. CrossRef Medline

Taylor KR, Holzer AK, Bazan JF, Walsh CA, Gleeson JG (2000) Patient mutations in doublecortin define a repeated tubulin-binding domain. J Biol Chem 275:34442-34450. CrossRef Medline

Toriyama M, Mizuno N, Fukami T, Iguchi T, Toriyama M, Tago K, Itoh H (2012) Phosphorylation of doublecortin by protein kinase A orchestrates microtubule and actin dynamics to promote neuronal progenitor cell migration. J Biol Chem 287:12691-12702. CrossRef Medline

Tsai JW, Bremner KH, Vallee RB (2007) Dual subcellular roles for LIS1 and dynein in radial neuronal migration in live brain tissue. Nat Neurosci 10:970-979. CrossRef Medline

Twery EN, Raper JA (2011) SDF1-induced antagonism of axonal repulsion requires multiple G-protein coupled signaling components that work in parallel. PLoS One 6:e18896. CrossRef Medline

Umeyama T, Okabe S, Kanai Y, Hirokawa N (1993) Dynamics of microtubules bundled by microtubule associated protein 2C (MAP2C). J Cell Biol 120:451-465. CrossRef Medline

Vallee RB, Tai C, Faulkner NE (2001) LIS1: cellular function of a diseasecausing gene. Trends Cell Biol 11:155-160. CrossRef Medline

Wang Y, Li G, Stanco A, Long JE, Crawford D, Potter GB, Pleasure SJ, Behrens T, Rubenstein JL (2011) CXCR4 and CXCR7 have distinct functions in regulating interneuron migration. Neuron 69:61-76. CrossRef Medline

Weaver AM, Karginov AV, Kinley AW, Weed SA, Li Y, Parsons JT, Cooper JA (2001) Cortactin promotes and stabilizes Arp2/3-induced actin filament network formation. Curr Biol 11:370-374. CrossRef Medline

Wells A, Huttenlocher A, Lauffenburger DA (2005) Calpain proteases in cell adhesion and motility. Int Rev Cytol 245:1-16. CrossRef Medline

Xu H, Leinwand SG, Dell AL, Fried-Cassorla E, Raper JA (2010) The calmodulin-stimulated adenylate cyclase ADCY8 sets the sensitivity of zebrafish retinal axons to midline repellents and is required for normal midline crossing. J Neurosci 30:7423-7433. CrossRef Medline 